\title{
The Crystal Structure of $\left[\left(\mathrm{UO}_{2}\right)_{2}(\mathrm{OH})_{2} \mathrm{Cl}_{2}\left(\mathrm{H}_{2} \mathrm{O}\right)_{4}\right]$
}

\author{
M ÄRTHA ÅBERG \\ Department of Inorganic Chemistry, Royal Institute of Technology (KTH), \\ S-100 44 Stockholm 70, Sweden
}

\begin{abstract}
The structure of the uranyl hydroxide chloride $\left[\left(\mathrm{UO}_{2}\right)_{2}(\mathrm{OH})_{2} \mathrm{Cl}_{2}\left(\mathrm{H}_{2} \mathrm{O}\right)_{4}\right]$ has been determined from three-dimensional $\mathrm{X}$-ray data. The crystals are monoclinic and the space group is No. 14: $P 2_{1} / n$. The unit cell contains four formula units (eight uranium atoms) and has the dimensions

$a=17.743 \AA, b=6.136 \AA, c=10.725 \AA$ and $\beta=95.52^{\circ}$

The structure is built up from discrete, uncharged molecules containing two linear uranyl groups, each of which is surrounded by one chlorine and four oxygen atoms forming an irregular planar pentagon. The two uranium atoms in a molecule are linked through a double $\mathrm{OH}$ bridge and the dimers are held together by hydrogen bonds.
\end{abstract}

The hydrolysis of the uranyl ion, $\mathrm{UO}_{2}{ }^{2+}$, has been studied repeatedly by 1 means of, e.g., emf methods and a list of papers on the subject published before 1964 is given in the IUPAC Tables of Stability Constants. ${ }^{1}$ The results indicate that a series of polynuclear complexes is formed. Two complexes, $\left(\mathrm{UO}_{2}\right)_{2}(\mathrm{OH})_{2}{ }^{2+}$ and $\left(\mathrm{UO}_{2}\right)_{3}(\mathrm{OH})_{5}{ }^{+}$, have come out with well-defined equilibrium constants in all ionic media, but additional complexes have also been suggested.

Emf data can at most give the charge and the number of metal atoms in the complex but cannot give any information about its atomic structure. A preliminary investigation of some hydroxide salts of uranium(VI), made by the author using X-ray methods, indicated that the compound $\mathrm{UO}_{3} \cdot \mathrm{HCl} \cdot 2 \mathrm{H}_{2} \mathrm{O}$, and the isomorphous bromide, both of which have previously been described in the literature, ${ }^{2,3}$ contain discrete dimers with a $U-U$ distance of about $3.9 \AA$. It seemed likely that this dinuclear complex would be similar to the one found in solution and a complete crystal structure determination has been performed.

\section{EXPERIMENTAL}

Preparation of crystals. The synthesis of $\mathrm{UO}_{3} \cdot \mathrm{HCl} \cdot 2 \mathrm{H}_{2} \mathrm{O}$ described by Mylius and Dietz ${ }^{2}$ was slightly modified. A solution of uranyl chloride was prepared by dissolving $\mathrm{UO}_{3}$ in concentrated hydrochloric acid. Sodium hydroxide was added until a precipitate

Acta Chem. Scand. 23 (1969) No. 3 
was formed. After filtration the solution was allowed to crystallize at room temperature. Thin yellow tabular crystals which were not quite stable outside the mother liquor appeared after some weeks.

Analysis. A weighed amount of the compound was dissolved in water and uranium was determined by precipitation with 8 -hydroxyquinoline according to a method described by Hecht and Donau." The amount of chloride was obtained by passing a measured volume of the solution of the dissolved crystals through a $\mathrm{H}^{+}$-saturated cation exchanger (Dowex $50 \times 8$ ) and titrating the eluate with standardized $\mathrm{NaOH}$. The water content was estimated by difference and was confirmed by the results of the structure investigation. The density was determined by comparison of the weight of a sample in benzene and in air. The results of the analysis are given in Table 1 and are also compared with calculated values for $\mathrm{UO}_{3} \cdot \mathrm{HCl} \cdot 2 \mathrm{H}_{2} \mathrm{O}$ assuming eight formula units in the unit cell.

\section{Table 1.}

Found

$\% \mathrm{UO}_{3}$
$\% \mathrm{HCl}^{\circ}$
$\% \mathrm{H}_{2} \mathrm{O}$
Density
79.8

10.4

9.8 (by difference)

4.05
Calculated for

$\mathrm{UO}_{3} \cdot \mathrm{HCl} \cdot 2 \mathrm{H}_{2} \mathrm{O}$

79.8

10.2

10.0

4.11

Crystals of the corresponding bromide, $\mathrm{UO}_{3} \cdot \mathrm{HBr} \cdot 2 \mathrm{H}_{2} \mathrm{O}$, were prepared according to a method described by Peterson. ${ }^{3}$ By comparison of Weissenberg photographs of the first five layer lines taken around the $b$ axis of both the chloride and bromide, the compounds were found to be isomorphous. Because of the lower atomic number of chlorine as compared with bromine, and a better stability, the compound $\mathrm{UO}_{3} \cdot \mathrm{HCl}_{2} \cdot 2 \mathrm{H}_{2} \mathrm{O}$ was chosen for the structure determination.

The calculations in connection with this investigation have been carried out on the CD 3600 computer in Uppsala and the IBM 7090 of FOA, Stockholm, using the programs described in Table 2.

Table 2. Computer programs used in the structure investigation.

\section{Program}

CELSIUS: Least squares refinement of unit cell dimensions

DATAP2: Lorentz-polarization and absorption corrections

DRF: Fourier summations and structure factor calculations

LALS: Least squares refinements of positional parameters and temperature factors

DISTAN: Interatomic distances and angles PLANE: Least squares fit of a plane to a set of points

LISTFC: Listing structure factor data (for IBM 7090)

\section{Authors}

J. Tegenfeldt, Uppsala, Sweden

P. Coppens, L. Leiserowitz and D. Rabinovich, revised by $M$. Elfström and $O$. Olofsson, Uppsala, Sweden

A. Zalkin, Berkeley, USA, modified by R. Liminga and J.-O. Lundgren, Uppsala, Sweden

P. K. Gantzel, R. A. Sparks and K. N. Trueblood, Los Angeles, USA, modified by A. Zalkin, Berkeley, USA and by C.-I. Brändén, R. Liminga and J.-O. Lundgren, Uppsala, Sweden

A. Zalkin, Berkeley, USA

C.-I. Brändén, Uppsala, Sweden

J. M. Stewart and R. L. Braun, University of Maryland, USA 


\section{UNIT CELL AND SPACE GROUP}

Rotation and Weissenberg photographs around the $b$ axis and precession photographs around the $a$ and $c$ axes showed that the crystals were monoclinic and gave approximate unit cell dimensions. In order to obtain more precise

Table 3. Powder photograph of $\mathrm{UO}_{3} \cdot \mathrm{HCl} \cdot 2 \mathrm{H}_{2} \mathrm{O}$ taken in a Guinier camera with $\mathrm{CuK \alpha}$ radiation. Values are given for the first lines only.

\begin{tabular}{|c|c|c|c|}
\hline$h k l$ & $10^{4} \sin ^{2} \theta_{\text {calc }}$ & $10^{4} \sin ^{2} \theta_{\mathrm{obs}}$ & $I_{\mathrm{obs}}$ \\
\hline 200 & 76 & 77 & $\mathrm{~s}$ \\
\hline 002 & $\left.\begin{array}{l}208 \\
210\end{array}\right\}$ & 210 & vs \\
\hline $\begin{array}{l}011 \\
210\end{array}$ & $\begin{array}{l}210 J \\
234\end{array}$ & 234 & vs \\
\hline$\overline{2} 02$ & 260 & 260 & vw \\
\hline 211 & 298 & 298 & $w$ \\
\hline 400 & 304 & 305 & $\mathrm{~s}$ \\
\hline 202 & 309 & 309 & vs \\
\hline 012 & 366 & 366 & $\mathrm{~s}$ \\
\hline 112 & 373 & 373 & vw \\
\hline$\overline{2} 12$ & 418 & 417 & vs \\
\hline 410 & 462 ) & & \\
\hline 402 & $464\}$ & 464 & vs \\
\hline 212 & $466)$ & & \\
\hline$\overline{4} 11$ & 490 & 489 & w \\
\hline 013 & 626 ) & 696 & $\mathbf{w}$ \\
\hline 020 & $630\}$ & 620 & $\mathbf{w}$ \\
\hline 113 & $663\}$ & 665 & vyw \\
\hline$\overline{2} 13$ & $666\}$ & 000 & Wow \\
\hline$\underline{6} 00$ & 685 & 685 & w \\
\hline$\overline{1} 21$ & 695 & 694 & w \\
\hline 220 & 706) & 706 & yyw \\
\hline 121 & $707\}$ & .00 & \\
\hline 412 & 719 & 720 & vw \\
\hline$\overline{2} 21$ & 746 & 746 & $\mathrm{~s}$ \\
\hline 221 & 771 & 771 & w \\
\hline$\overline{6} 02$ & 820 & 820 & $\mathrm{~m}$ \\
\hline$\underline{0} 04$ & 833) & 834 & \\
\hline$\overline{3} 21$ & $835\}$ & 834 & $\mathrm{~m}$ \\
\hline$\overline{4} 13$ & $858\}$ & & \\
\hline$\overline{\mathbf{6}} 11$ & $858\}$ & 858 & $\mathrm{~m}$ \\
\hline$\overline{2} 04$ & $861)$ & & \\
\hline 122 & 870 & 872 & vvw \\
\hline 321 & $872\}$ & & vVw \\
\hline 222 & 939 & 940 & vvw \\
\hline 204 & 958 & 958 & $\mathrm{~m}$ \\
\hline$\overline{4} 21$ & 963) & 965 & $\mathrm{w}$ \\
\hline 602 & $966\}$ & & \\
\hline$\overline{6} 12$ & 978 & 977 & $\mathrm{~m}$ \\
\hline 014 & 991 & 990 & $\mathrm{~s}$ \\
\hline$\overline{5} 13$ & $1011\}$ & 1013 & $\mathrm{~m}$ \\
\hline 421 & 1011\} & 1030 & \\
\hline$\overline{4} 04$ & 1040 & 1039 & $\mathrm{~s}$ \\
\hline 023 & $1099\}$ & 1098 & s \\
\hline $\begin{array}{l}\frac{1}{2} 23 \\
\overline{2} 23\end{array}$ & $\begin{array}{l}1100 f \\
1139\end{array}$ & 1137 & $\mathrm{~s}$ \\
\hline
\end{tabular}

Acta Chem. Scand. 23 (1969) No. 3 
values of the lattice parameters, a powder photograph was taken with $\mathrm{Cu} K \alpha$ radiation $(\lambda=1.5405 \AA)$ in a Guinier focusing camera with $\mathrm{KCl}(a=6.29294 \AA$ at $25^{\circ} \mathrm{C}$ ) as internal standard (Table 3 ). By means of a least squares refinement (unit weights) the unit cell was found to have the following dimensions with maximum errors $3 \sigma$.*

$$
a=17.743 \pm 7 \AA, b=6.136 \pm 2 \AA, c=10.725 \pm 3 \AA \text { and } \beta=95.52 \pm 3^{\circ}
$$

The corresponaing values for the bromide were approximately

$$
a=18.1_{7} \AA, b=6.3_{7} \AA, c=10.9_{1} \AA \text { and } \beta=97^{\circ}
$$

Using the multiple film technique, Weissenberg photographs were taken around the $b$ axis ( 0 to 6 th layer line) with $\operatorname{Mo} K \alpha$ radiation $(\lambda=0.7107 \AA$ ) and around the $c$ axis ( 0 to 6 th layer line) with $\mathrm{Cu} K \alpha$ radiation $(\lambda=1.5418$ $\AA)$. Because the crystals were found to be slightly deliquescent, they had to be placed in thin-walled glass capillaries for these experiments.

The systematic absences were such that

$h 0 l$ occurred only for $h+l=2 n$

$0 k 0 "$ " " $k=2 n$

This is characteristic of space group No. 14: $P 2_{1} / n$ (the setting differs from that given in the International Tables ${ }^{5}$ ).

Intensities were estimated visually by comparison with calibrated intensity scales and were corrected for Lorentz and polarization factors and for absorption. The data which were used for the absorption corrections are summarized

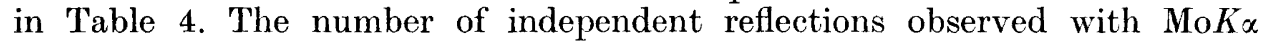

Table 4. Data used for the absorption corrections of $\mathrm{UO}_{3} \cdot \mathrm{HCl} \cdot 2 \mathrm{H}_{2} \mathrm{O}$. The $b$ axis is orientated in the long direction of the crystal plate and the $c$ axis along the diagonal of the cross section.

\begin{tabular}{ccccc}
\hline Rotation axis & Radiation & $\begin{array}{c}\text { Crystal length } \\
(\mathrm{mm})\end{array}$ & $\begin{array}{c}\text { Cross section } \\
(\mathrm{mm} \times \mathrm{mm})\end{array}$ & $\begin{array}{c}\text { Absorption } \\
\text { coefficient }\left(\mathrm{cm}^{-1}\right)\end{array}$ \\
\hline$b$ & $\mathrm{MoK \alpha}$ & 0.324 & $0.051 \times 0.122$ & 338 \\
$c$ & $\mathrm{CuK \alpha}$ & 0.113 & $0.021 \times 0.055$ & 1104 \\
\hline
\end{tabular}

radiation was 2474 , corresponding to about $60 \%$ of those with $\sin \theta / \lambda \leqq 1$. Reflections with a value of $\sin \theta / \lambda \gtrsim 1$ were generally too weak to be observed. The number of intensity values recorded around the $c$ axis was 1112 , which were about $70 \%$ of those within the $\mathrm{CuK \alpha}$ sphere.

\section{STRUCTURE DETERMINATION}

The structure determination was based on the data taken with MoKa radiation around the $b$ axis.

* All standard deviations and maximum errors are given as errors in the last figure of the corresponding value. 
Preliminary scale factors for putting the intensities of the reflections of the seven layers on an absolute scale were obtained using Wilson's method. ${ }^{6}$ In order to find the positions of the uranium atoms, a three-dimensional Patterson function, $\mathrm{P}(u, v, w)$, was then calculated. Table 5 lists those peaks

Table 5. Peaks $(>100)$ in the three-dimensional Patterson function (expected height for a single $\mathrm{U}-\mathrm{U}$ vector is 120 ).

\begin{tabular}{lllcc}
\hline$u$ & $v$ & $w$ & Peak height & $\begin{array}{c}\text { Number of vectors } \\
\text { U-U }\end{array}$ \\
\hline 0.50 & 0.09 & 0 & 492 & 4 \\
0.50 & 0.12 & 0.50 & 249 & 2 \\
0.31 & 0.195 & 0.095 & 119 & 1 \\
0 & 0.215 & 0.50 & 468 & 4 \\
0.185 & 0.285 & 0.905 & 222 & 2 \\
0.50 & 0.31 & 0.50 & 259 & 2 \\
0.32 & 0.375 & 0.09 & 115 & 1 \\
0.315 & 0.40 & 0.595 & 228 & 2 \\
0.185 & 0.50 & 0.405 & 418 & 4 \\
\hline
\end{tabular}

which have a height larger than 100 on a scale where the expected height is 120 for a single $U-U$ vector.

From the values of $u, v$, and $w$ in Table 5 it is obvious that the eight uranium atoms must occupy two of the general fourfold positions: $\pm(x, y, z)$; $\pm\left(\frac{1}{2}+x, \frac{1}{2}-y, \frac{1}{2}+z\right)$ in space group $P 2_{1} / n$.

On the hol photographs all reflections with $l$ odd are extremely weak and it seems likely that the uranium atoms make very small contributions to the intensities of these reflections. Accordingly the two independent sets of uranium atoms must be approximately related by a glide plane perpendicular to the $b$ axis and with the glide direction parallel to the $c$ axis. This leads to the following relations between the parameters for the two sets of uranium atoms

$4 \mathrm{U}(1)$ in $4(e): \pm\left(x, y_{1}, z\right) ; \pm\left(\frac{1}{2}+x, \frac{1}{2}-y_{1}, \frac{1}{2}+z\right)$;

$4 \mathrm{U}(2)$ in $4(e): \pm\left(x, y_{2}, \frac{1}{2}+z\right)$; $\pm\left(\frac{1}{2}+x, \frac{1}{2}-y_{2}, z\right)$;

The vectors between these positions are

$$
\begin{aligned}
& \frac{1}{2}, \frac{1}{2}-2 y_{1}, \frac{1}{2} \\
& \frac{1}{2}, \frac{1}{2}-2 y_{2}, \frac{1}{2} \\
& \frac{1}{2}-2 x, \frac{1}{2}, \frac{1}{2}-2 z \\
& 2 x, \pm 2 y_{1}, 2 z \\
& 2 x, \pm 2 y_{2}, 2 z \\
& 0, \pm\left(y_{1}-y_{2}\right), \frac{1}{2} \\
& \frac{1}{2}, \frac{1}{2} \pm\left(y_{1}+y_{2}\right), 0 \\
& 2 x, \pm\left(y_{1}+y_{2}\right), \frac{1}{2}+2 z \\
& \frac{1}{2}+2 x, \frac{1}{2}+\left(y_{1}-y_{2}\right), 2 z
\end{aligned}
$$$$
\left.\begin{array}{cc}
\text { weight } & 2 \\
» & 2 \\
» & 4 \\
》 & 1 \\
》 & 1
\end{array}\right\}
$$

within the fourfold positions

weight 4

$\left.\begin{array}{ll}\text { " } & 4 \\ » & 2 \\ » & 2\end{array}\right\}$

between the fourfold positions

When comparing these vectors with the values of $u, v$ and $w$ in Table 5 it can be shown that the only possible combinations of $x$-, $y$ - and $z$-parameters

Acta Chem. Scand. 23 (1969) No. 3 
$\left(0 \leq x \leq \frac{1}{2}, 0 \leq y \leq \frac{1}{2}, 0 \leq z \leq 1\right)$ are $x=0.16 ; y=0.09,0.19,0.31$ or $0.41 ; z=0.05$ or 0.55 or $x=0.34 ; y=0.09,0.19,0.31$ or $0.41 ; z=0.45$ or 0.95 .

The parameters of the first set of uranium atoms which can be chosen arbitrarily from the above combinations have been given the following values $x_{1}=0.16 ; y_{1}=0.31 ; z_{1}=0.05$.

Now the choice of the parameters of the second set of uranium atoms is restricted to the values $x_{2}=0.16 ; y_{2}=0.09 ; z_{2}=0.55$ by the approximate glide plane relation and by the peaks in the Patterson function. Thus the glide plane perpendicular to the $b$ axis is situated at $y \approx 0.20$.

All vectors within and between these two fourfold positions are present as peaks in the three-dimensional Patterson function and have approximately the heights expected from the weights of the corresponding vectors (Table 5).

The parameters of the uranium atoms were now subjected to a full-matrix least squares refinement. Individual scale factors for the seven layers and isotropic temperature factors were also refined. The following values were obtained: $x_{1}=0.1606 ; y_{1}=0.3150 ; z_{1}=0.0468$ and $x_{2}=0.1543 ; y_{2}=0.1009$; $z_{2}=0.5487$.

The reliability index, defined by $R=\sum\left|F_{\mathrm{o}}-\right| F_{\mathrm{c}} \mid / \sum F_{\mathrm{o}}$, was lowered from 0.292 to 0.189 in four cycles.

To locate the light atoms, an electron density map based on $F_{\mathrm{o}}-F_{\mathrm{c}} \mathrm{U}$ was calculated. The $F_{\mathrm{o}}$ values were given the signs of the structure factors for the uranium atoms obtained from the above coordinates $\left(F_{\mathrm{c}} \mathrm{U}\right)$. All the chlorine and oxygen atoms which occupy the general fourfold position $4(e)$ were found

Table 6. Final positional and thermal parameters based on the MoK$\alpha$ data. The positional parameters given belong to atoms in the same dimer. Standard deviations $(\sigma)$ are given in parentheses. All atoms are in the positions $4(e)( \pm(x, y, z)$; $\pm\left(\frac{1}{2}+x, \frac{1}{2}-y, \frac{1}{2}+z\right)$;) in space group No. $14: P 2_{1} / n$.

\begin{tabular}{lllll} 
Atom & \multicolumn{1}{c}{$x$} & \multicolumn{1}{c}{$y$} & \multicolumn{1}{c}{$z$} & $B$ or $B_{11}$ \\
& & & & \\
$\mathrm{U}(1)$ & $0.33919(5)$ & $0.31520(16)$ & $0.45281(8)$ & $0.49(2)$ \\
$\mathrm{U}(2)$ & $0.15424(5)$ & $0.60167(16)$ & $0.54829(8)$ & $0.55(2)$ \\
$\mathrm{Cl}(1)$ & $0.4170(4)$ & $0.5963(13)$ & $0.3113(7)$ & $1.5(2)$ \\
$\mathrm{Cl}(2)$ & $0.0787(4)$ & $0.3241(14)$ & $0.6943(7)$ & $2.1(2)$ \\
$\mathrm{O}(1)$ & $0.2659(12)$ & $0.6293(39)$ & $0.4411(20)$ & $1.7(3)$ \\
$\mathrm{O}(2)$ & $0.2282(11)$ & $0.2834(35)$ & $0.5623(18)$ & $1.3(3)$ \\
$\mathrm{O}(3)$ & $0.2842(11)$ & $0.2022(36)$ & $0.3157(19)$ & $1.6(3)$ \\
$\mathrm{O}(4)$ & $0.3897(8)$ & $0.4222(29)$ & $0.5874(14)$ & $0.6(2)$ \\
$\mathrm{O}(5)$ & $0.1012(11)$ & $0.4895(37)$ & $0.4102(18)$ & $1.5(3)$ \\
$\mathrm{O}(6)$ & $0.2073(11)$ & $0.7171(36)$ & $0.6790(18)$ & $14(3)$ \\
$\mathrm{O}(7)$ & $0.3478(10)$ & $-0.0400(35)$ & $0.5390(17)$ & $1.2(3)$ \\
$\mathrm{O}(8)$ & $0.4520(11)$ & $0.1044(36)$ & $0.3897(19)$ & $1.5(3)$ \\
$\mathrm{O}(9)$ & $0.1517(9)$ & $0.9617(33)$ & $0.4591(16)$ & $0.9(2)$ \\
$\mathrm{O}(10)$ & $0.0425(18)$ & $0.7995(53)$ & $0.6082(30)$ & $3.7(6)$
\end{tabular}

\begin{tabular}{cllllr} 
& \multicolumn{1}{c}{$B_{22}$} & \multicolumn{1}{c}{$B_{33}$} & \multicolumn{1}{c}{$B_{12}$} & \multicolumn{1}{c}{$B_{13}$} & \multicolumn{1}{c}{$B_{23}$} \\
$\mathrm{U}(1)$ & $0.65(5)$ & $0.81(3)$ & $0.09(4)$ & $0.23(4)$ & $0.03(5)$ \\
$\mathrm{U}(2)$ & $0.65(5)$ & $0.74(3)$ & $-0.05(5)$ & $0.21(4)$ & $-0.25(5)$ \\
$\mathrm{Cl}(1)$ & $2.1(4)$ & $2.3(3)$ & $-0.2(4)$ & $0.9(4)$ & $0.8(4)$ \\
$\mathrm{Cl}(2)$ & $2.5(4)$ & $1.7(3)$ & $-1.1(4)$ & $1.4(4)$ & $0.1(4)$
\end{tabular}


Table \%. Final observed and calculated structure factors of $\mathrm{UO}_{3} \cdot \mathbf{H C l} \cdot 2 \mathrm{H}_{2} \mathrm{O}$. The columns are $h, 10\left|F_{\mathrm{o}}\right|$, and $10 F_{\mathrm{c}}$. Unobserved reflections are marked with an asterisk and have been given $F_{\mathrm{o}}$ values corresponding to the estimated minimum observable intensity. Values marked with a plus have been taken from the $\mathrm{Cu} K \alpha$ data. All other values are MoK$\alpha$ data.

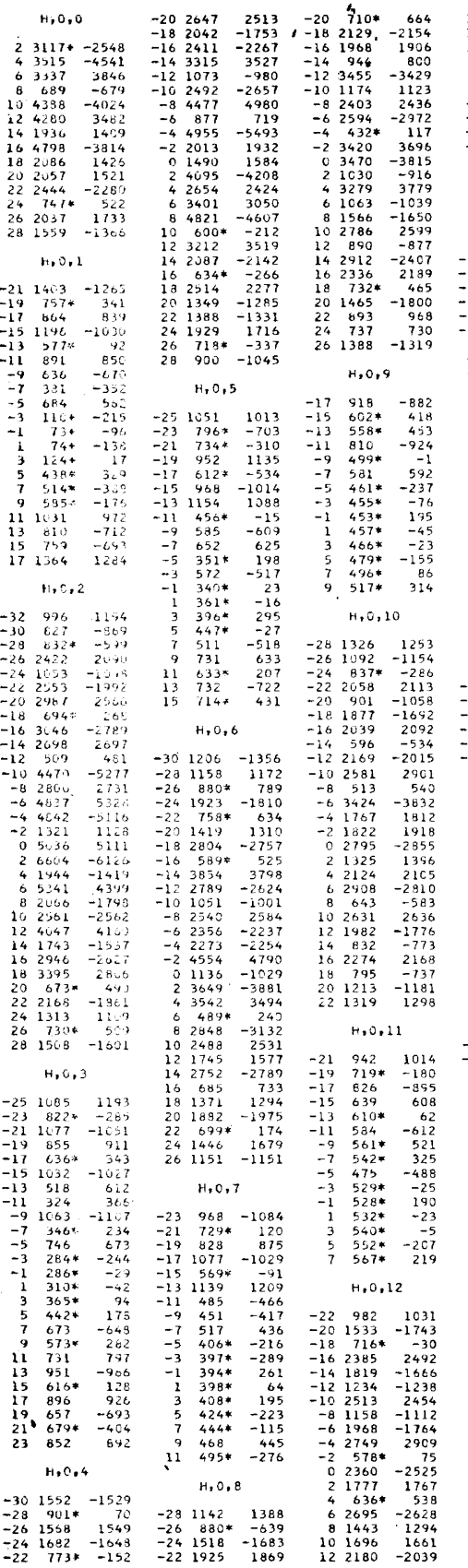

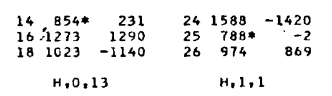

$\begin{array}{llllll}-7 & 623 * & -55 & -26 & 1172 & 1205 \\ -5 & 616 * & -331 & -25 & 830 * & -442 \\ -3 & 612 * & 256 & -24 & 835 * & -426\end{array}$

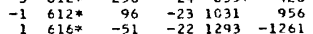

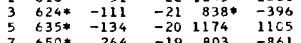

H, 0,14

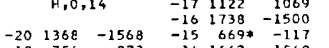

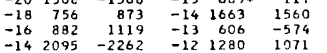

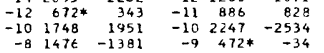

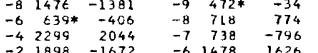

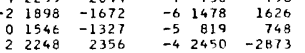

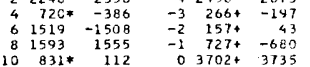

$\begin{array}{rrr}10 & 831 * & 112 \\ 12 & 1518 & -1502 \\ 14 & 154 & 1033\end{array}$

$H, 0,15$

$\begin{array}{ccc}-5 & 682 * & -119 \\ -3 & 679 * & 177\end{array}$

$\begin{array}{rrr}-1 & 679 * & 177 \\ -1 & 680 * & -118 \\ 1 & 684 * & -76 \\ 3 & 691 * & -4 \\ 5 & 901 * & 7\end{array}$

H.C,16

$\begin{array}{rrr}-20 & 1008 & -1315 \\ -18 & 974 & 1218 \\ -18 & 770 & 180\end{array}$

$\begin{array}{lll}-18 & 974 & 1218 \\ -16 & 776 * & 180 \\ -14 & 1180 & -1234 \\ -12 & 1156 & -1265\end{array}$

$\begin{array}{rrr}-14 & 1186 & -1234 \\ -12 & 1156 & 1265 \\ -10 & 724 * & 583 \\ -8 & 1774 & -1933\end{array}$

$\begin{array}{rrr}-14 & 724 * & 583 \\ -8 & 1774 & -2933 \\ -6 & 716 * & 509 \\ -6 & 132 & -364\end{array}$

$\begin{array}{rrr}-4 & 1324 & 1344 \\ -2 & 1602 & -1720\end{array}$

$\begin{array}{lll}0 & 749 * & 279 \\ 2 & 1699 & 1491\end{array}$

$\begin{array}{lll}2 & 1140 & -1206 \\ 6 & 847 * & -488 \\ 8 & 1223 & 1378\end{array}$

$\mathrm{H}, 0,17$

$\begin{array}{ccc}-3 & 743 * & 181 \\ -1 & 744 * & -84 \\ 2 & 747 * & -4\end{array}$

\begin{tabular}{rrr}
1 & $747 *$ & -84 \\
3 & $753 *$ & -4 \\
\hline
\end{tabular}

$$
\text { H.0, } 18
$$

$\begin{array}{llll}-12 & 971 & 1198 & -\end{array}$

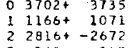

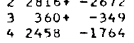

$\begin{array}{rrr}5 & 554 & -423 \\ 6 & 2613 & 2259 \\ 7 & 618 & 637 \\ 8 & 615 & 630\end{array}$

$\begin{array}{rrrr}8 & 618 & 637 \\ 8 & 454 & -460 \\ 9 & 600 & -659 \\ 10 & 1066 & -1021 \\ 11 & 1021\end{array}$

$\begin{array}{lrr}10 & 1066 & -1021 \\ 11 & 684 * & -521 \\ 12 & 2917 & 2474\end{array}$

$\begin{array}{lll}12 & 2917 & 2474 \\ 13 & 1369 & 1131 \\ 14 & 696 * & -287 \\ 16 & 896 & -284\end{array}$

$\begin{array}{lll}14 & 696 * & -287 \\ 15 & 697 * & -394 \\ 16 & 2463 & -2146\end{array}$

$\begin{array}{lll}176 & 243 & -2146 \\ 17 & 708 & -642 \\ 11 & 1080 & 153\end{array}$

$\begin{array}{ccc}18 & 1080 & 1053 \\ 19 & 1014 & 1019 \\ 20 & 649 & 722\end{array}$

$\begin{array}{lll}21 & 733 * & -289 \\ 22 & 1503 & -1231 \\ 23 & 752 & -617\end{array}$

$\begin{array}{llll}23 & 752 & -677\end{array}$

$H, 1,2$

$\begin{array}{lll}-28 & 1223 & -1116 \\ -27 & 870 * & -657 \\ -26 & 879 * & -655\end{array}$

$\begin{array}{ccc}-26 & 879 * & -405 \\ -25 & 890 * & 386 \\ -25 & 1890 * & 268\end{array}$

$\begin{array}{lll}-24 & 1688 & 1614 \\ -23 & 680 * & 488 \\ -22 & 1050\end{array}$

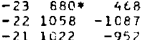

$\begin{array}{lll}-21 & 1022 & -952 \\ -20 & 797 * & -543 \\ -19 & 760 * & -82 \\ -18 & 2916 & 2389 \\ -17 & 2102 & -1259\end{array}$

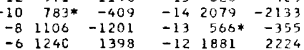

$\begin{array}{lll}-17 & 1202 & 1055\end{array}$

$\begin{array}{lll}-4 & 794 * & 287 \\ -2 & 1273 & -1355 \\ -0 & 1275\end{array}$

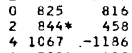

$\begin{array}{rrrr}4 & 1067 * & -1186 \\ 8 & 900 * & 489 \\ 8 & 836 & 932\end{array}$

$\mathrm{H}, 1.0$

$\begin{array}{rrr}1 & 852+ & -750 \\ 2 & 447 t+ & 3985\end{array}$

$\begin{array}{ccc}2 & 8576+ & -790 \\ 3 & 4475 \\ 3 & 975 & -1078 \\ 4 & 2918 & -3797 \\ 6 & 593 & -994\end{array}$

$\begin{array}{ccc}4 & 2918 & -3797 \\ 5 & 399 * & -156 \\ 6 & 583 & -934 \\ 7 & 1065 & -1039 \\ 9 & 3950 & 4286\end{array}$

$\begin{array}{rrr}7 & 1065 & -1039 \\ 8 & 3830 & 4836 \\ 9 & 771 & 707\end{array}$

$\begin{array}{rrr}10 & 1331 & -1168 \\ 11 & 906 & 797 \\ 12 & 2009 & -1602 \\ 11 & 2094 & 2168\end{array}$

$\begin{array}{lll}11 & 906 & 798 \\ 12 & 2009 & -1602 \\ 13 & 834 & -807 \\ 14 & 264 t & 2168 \\ 15 & 264 & 2161\end{array}$

$\begin{array}{lll}14 & 264 t & 2168 \\ 15 & 739 * & -111 \\ 16 & 763 & -720\end{array}$

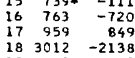

$\begin{array}{lll}18 & 3012 & -2138 \\ 19 & 783 * & -602 \\ 20 & 2612 & 2018\end{array}$

$\begin{array}{rrr}20 & 2612 & 2018 \\ 21 & 776 * & -462 \\ 22 & 775 * & 300 \\ 23 & 697 & 769\end{array}$

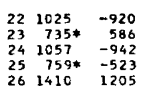

$\mathrm{H}, 1,3$

$\begin{array}{lll}-26 & 844 & 855 \\ -25 & 927 * & -5\end{array}$

$\begin{array}{ccc}-24 & 1105 & -1123 \\ -23 & 868 * & -5 \\ -22 & 851\end{array}$

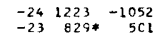

$\begin{array}{rrrrrr}-20 & 2164 & 1670 & -22 & 716 & 667 \\ -19 & 732 * & -33 & -21 & 770 & -877\end{array}$

$\begin{array}{rlllllr}-18 & 698 * & -587 & -20 & 1043 & 1006 \\ -17 & 870 & 1007 & -19 & 708 * & 383 \\ -15 & 668 & -56 & -19 & 643 & 428\end{array}$

$\begin{array}{rrrrrr}-16 & 1026 & -1032 & -18 & 1851 & -1933 \\ -15 & 663 & -658 & -17 & 643 * & 428\end{array}$

$\begin{array}{llllll}-14 & 1761 & 1921 & -16 & 647 * & -169 \\ -13 & 543 * & -311 & -15 & 1097 & -1692\end{array}$

$\begin{array}{rrrrrr}12 & 1012 & -1080 & -14 & 2171 & 2339 \\ -11 & 762 & 732 & -13 & 529 * & 338 \\ -11 & 2615 & -222 & -12 & 1354 & -1551\end{array}$

$\begin{array}{lllllll}10 & 2115 & -2227 & -13 & 529 * & 338 \\ -12 & 1354 & -1551 \\ -9 & 657 & -661 & -11 & 678 & 848\end{array}$

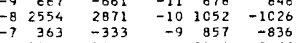

$\begin{array}{llllll}-7 & 363 & -333 & -9 & 857 & -836 \\ -6 & 1052 & 1068 & -8 & 2164 & 2452\end{array}$

$\begin{array}{rrrrrr}-5 & 1135 & 1194 & -7 & 396 * & 128 \\ -4 & 3016 & -3459 & -6 & 1138 & -1180 \\ -3 & 715 & -610 & -5 & 518 & 510 \\ -2 & 164 & -610 & -4 & 238 & -240\end{array}$

$\begin{array}{cccccc}-3 & 715 & -610 & -5 & 518 & -510 \\ -2 & 1074 & 973 & -4 & 2237 & -24 c 0 \\ -1 & 024 & -305 & -3 & 1265 & -130\end{array}$

$\begin{array}{rrrrrr}-1 & 261 & -365 & -3 & 1065 & -1 c 06 \\ 0 & 1369 & 1347 & -2 & 2831 & 2214 \\ 1 & 647 & 519 & -1 & 343 & 341 \\ 2 & 245 & -239 & -1 & 349 & 31\end{array}$

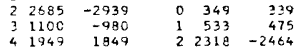

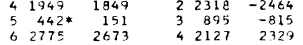

$\begin{array}{llllll}7 & 1325 & 898 & 5 & 798 & 676 \\ 8 & 2885 & -2381 & 6 & 981 & 839 \\ 9 & 998 & -889 & 7 & 534 * & 370\end{array}$

1c $1008-809 \quad 8 \quad 3043-3157$

$\begin{array}{rrrrrr}11 & 63 C * & 116 & 9 & 928 & -836 \\ 17 & 1978 & 22.12 & 10 & 682 * & 555\end{array}$

$\begin{array}{rrrrrr}13 & 935 & 892 & 11 & 167 * & 362 \\ 14 & 1353 & -1368 & 12 & 1857 & 1951 \\ 15 & 1220 & -1249 & 14 & 1654 & -159\end{array}$

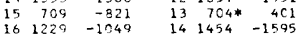

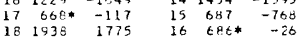

$\begin{array}{llllll}19 & 841 & 819 & 17 & 688 * & 384 \\ 20 & 605 * & -171 & 18 & 1275 & 3295\end{array}$

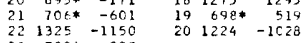

$23 \quad 732 *-23$

$\begin{array}{lll}24 & 905 & 905 \\ 25 & 917 & 844\end{array}$

$\mathrm{H}, 1,4$

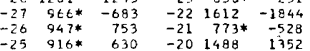

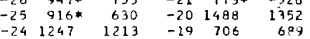

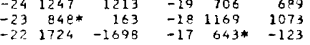

$\begin{array}{rrrrrr}-21 & 776 * & -687 & -16 & 2269 & -2153 \\ -20 & 74 \% * & 157 & -15 & 836 & -912 \\ -19 & 1604 & 1542 & -13 & 659 & -263\end{array}$

$\begin{array}{rrrrrr}-19 & 717 * & 600 & -14 & 1142 & 1021 \\ -13 & 1604 & 1442 & -13 & 659 & 703 \\ -116 & 2654 & -2078 & -11 & 64 & 618\end{array}$

$\begin{array}{llllll}-17 & 655 * & 509 & -12 & 1484 & 1570\end{array}$

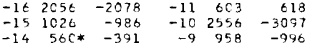

$\begin{array}{rrrrrrr}-14 & 566 * & -391 & -9 & 958 & -996 \\ -13 & 531 * & -43 & -8 & 717 & -962 \\ -12774 & 3398 & -7 & 417 * & -79 \\ -11 & 939 & 923 & -6 & 2734 & 3098 \\ -11 & 939 & 923 & -6 & 2734 & 3098\end{array}$

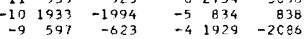

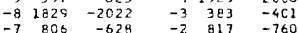

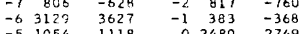

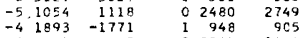

$\begin{array}{lllllll}-3 & 311 & -325 & 2 & 2237 & -2486 \\ -2 & 1356 & -1421 & 3 & 432 * & 41\end{array}$

$\begin{array}{rrrrrr}-1 & 814 & -833 & 4 & 2099 & -2156 \\ 0 & 3229 & 3653 & 5 & 1134 & -1196 \\ 1 & 634 & 623 & 6 & 63293 & 3648 \\ 2 & 166 & 843 & 6 & 363\end{array}$

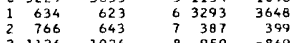

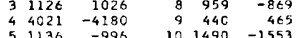

$\begin{array}{rrrrrr}4 & 4021 & -4180 & 9 & 44 C & 465 \\ 5 & 1136 & -996 & 10 & 1490 & -1553 \\ 6 & 2390 & 2225 & 11 & 712 * & -553\end{array}$

$\begin{array}{rrrrrr}7 & 536 * & -186 & 12 & 2034 & 19 C 3 \\ 8 & 593 * & 478 & 13 & 738 * & -57\end{array}$

$\begin{array}{rrrrrr}9 & 808 & 784 & 14 & 727 * & -49 \\ 10 & 3046 & -3040 & 15 & 717 & 697\end{array}$

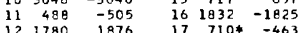

$\begin{array}{llllll}12 & 1780 & 1876 & 17 & 710 * & -463 \\ 13 & 659 * & -513 & 18 & 1308 & 1391 \\ 13 & 1390 & 1508 & 198 & 718 * & -412\end{array}$

$\begin{array}{rrrrrr}14 & 1490 & 1468 & 19 & 718 * & -412 \\ 15 & 720 & 762 & 20 & 720 & 632 \\ 16 & 2121 & -2081 & 21 & 727 & 719\end{array}$ 
Table 7. Continued.

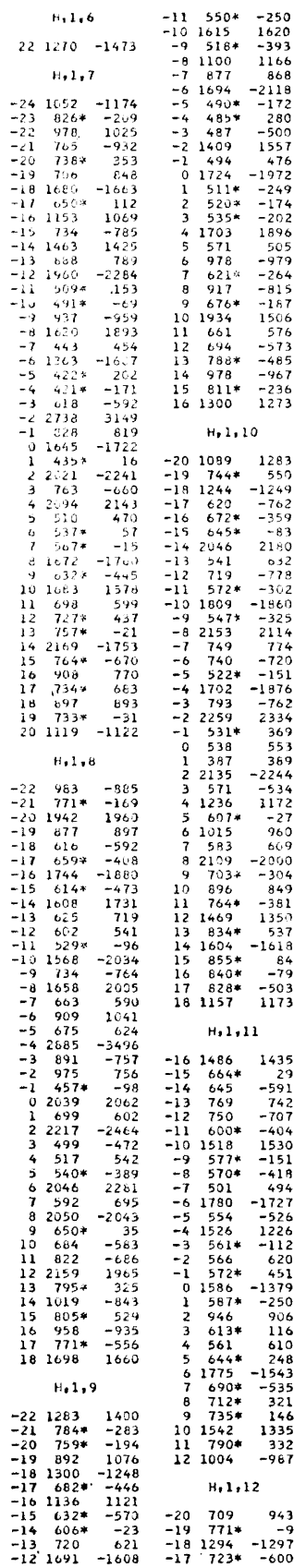

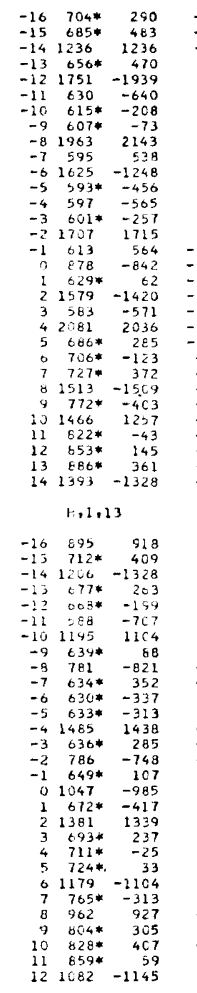

$$
\mathrm{H}, \mathrm{i}, 14
$$

$\begin{array}{lll}-18 & 1245 & -1440 \\ -17 & 767 * & -320 \\ -16 & 762 * & \end{array}$

$\begin{array}{lll}-17 & 767 * & -320 \\ -16 & 921 & 933 \\ -15 & 739 * & 573 \\ -14 & 73 \% & \end{array}$ $\begin{array}{rrr}-14 & 722 * & 556 \\ -13 & 714 * & -75 \\ -12 & 2290 & -1384 \\ -11 & 690 * & -686\end{array}$ $\begin{array}{lll}-13 & 714 * & -75 \\ -12 & 1290 & -1384 \\ -11 & 690 * & -646\end{array}$ $\begin{array}{rrr}-10 & 836 * & 967 \\ -9 & 677 * & 318 \\ -7 & 623 * & 363\end{array}$ $\begin{array}{lll}-8 & 823 & 883 \\ -7 & 667 * & 363\end{array}$ $\begin{array}{lrr}-6 & 1829 & -1716 \\ -5 & 667 * & -443 \\ -4 & 672 * & 212\end{array}$ $\begin{array}{rrr}-4 & 672 * & 212 \\ -3 & 678 * & 6 \\ -2 & 1629 & 1417\end{array}$ $\begin{array}{ccc}-2 & 1629 & 1417 \\ -1 & 692 * & 446 \\ 0 & 1 & 24\end{array}$ $\begin{array}{ccc}-1 & 692 * & 446 \\ 0 & 1424 & -1282 \\ 1 & 1426 & -283\end{array}$ $\begin{array}{lll}1 & 716 * & -243 \\ 2 & 726 * & -176 \\ 3 & 737 * & -277 \\ 4 & 732 * & -275\end{array}$

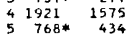
$\begin{array}{rrr}1243 & -1199 \\ 7 & 803 * & -18 \\ 6 & 737 & -612\end{array}$ $\begin{array}{rrrr}8 & 737 & -612 \\ 9 & 848 * & -393 \\ 9 & 8406 & 1213\end{array}$

$$
\mathrm{H}, 1,15
$$

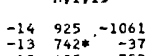
$\begin{array}{rrrr}-13 & 742 * & -37 \\ -12 & 655 & 752 \\ -11 & 728 * & -330 \\ -10 & 042 & -321\end{array}$ $\begin{array}{rrr}-11 & 728 * & -330 \\ -10 & .642 & 621 \\ -9 & 716 * & 463 \\ -0 & 7228 & -2139\end{array}$ $\begin{array}{rrr}-8 & 1228 & -1139 \\ -7 & 707 * & 45 \\ -6 & 711 * & 299\end{array}$ $\begin{array}{rrr}-7 & 707 * & 45 \\ -6 & 711 * & 299 \\ -5 & 708 * & -342 \\ -4 & 873 & 926\end{array}$

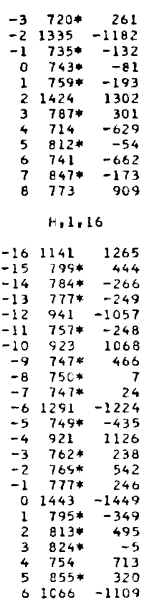

$\mathrm{H}, 1,17$

$\begin{array}{lll}-8 & 960 & -1021 \\ -7 & 785 * & -283 \\ -6 & 78, & -830\end{array}$

$\begin{array}{rrr}-6 & 783 & 830 \\ -5 & 788 * & -156 \\ -4 & 795 * & 531\end{array}$

$\begin{array}{lll}-5 & 788 * & -156 \\ -4 & 795 * & 531 \\ -3 & 802 * & 326 \\ -2 & 326 & -103\end{array}$

$\begin{array}{rrr}-2 & 986 & -1023 \\ -1 & 818 * & -102\end{array}$

$\begin{array}{lll}0 & 827 * & 362 \\ 1 & 836 * & -70 \\ 2 & 854 * & 583\end{array}$

$\begin{array}{lll}3 & 865 * & 172 \\ 4 & 789 & -904 \\ & & \end{array}$

$\mathrm{H}, 1,18$

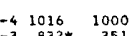

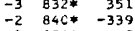

$\begin{array}{rrrr}-2 & 846 * & -339 \\ -1 & 856 * & -5 \\ 0 & 773 & -549 \\ 1 & 874 * & -332 \\ 2 & 874 & 935 \\ 2 & 791 & 935\end{array}$

$$
\mathrm{H}, 2, \mathrm{O}
$$

O $2425+-1201$

$\begin{array}{rrr}1 & 675+ & -588 \\ 2 & 1038+ & 900 \\ 3 & 232+ & \end{array}$

$\begin{array}{rrr}3 & 232 * & 8 \\ 4 & 533 & -429 \\ 5 & 378 * & 167\end{array}$

$\begin{array}{rrr}5 & 378 * & -429 \\ 6 & 695 & -702 \\ 7 & 450 * & 150\end{array}$

$\begin{array}{rlr}7 & 450 * & 150 \\ 8 & 81 \mathrm{C} & 735 \\ 9 & 782 & -700 \\ 9 & 547 & 394\end{array}$

$\begin{array}{lll}10 & 582 & -700 \\ 11 & 547 * & 394 \\ 11 & 577 * & 321 \\ 12 & 748 & -662 \\ 14 & 672 * & 126\end{array}$

$\begin{array}{rlr}12 & 748 & -662 \\ 13 & 788 & 720 \\ 14 & 672 * & 116 \\ 15 & 915 & -906\end{array}$

$\begin{array}{lll}15 & 672 * & 116 \\ 15 & 915 & -906 \\ 16 & 723 * & 519 \\ 17 & 731 * & -118 \\ 19 & 909 & -740\end{array}$

$\begin{array}{ccc}18 & 909 & -740 \\ 19 & 1042 & 1041 \\ 20 & 730 * & 361\end{array}$

$\begin{array}{lll}21 & 731 * & -406 \\ 22 & 730 * & 487 \\ 23 & 732 * & -538\end{array}$

$\begin{array}{lll}24 & 732 * & -538 \\ 25 & 664 & -717 \\ & 604\end{array}$

$$
\mathrm{H}, 2,1
$$

$\begin{array}{lll}-30 & 957 & 1087\end{array}$

$\begin{array}{rrr}-30 & 957 & 1087 \\ -29 & 699 & 666 \\ -28 & 955 & -1028 \\ -27 & 956 & -208\end{array}$

$\begin{array}{lll}-27 & 780 * & 108 \\ -26 & 779 * & -392 \\ -25 & 798 & -167\end{array}$

$\begin{array}{lll}-26 & 779 * & -392 \\ -25 & 782 & -767 \\ -24 & 1716 & 1574 \\ -23 & 793\end{array}$

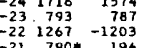

$\begin{array}{lll}-22 & 1267 & -1203 \\ -21 & 790 * & 194 \\ -20 & 1234 & -1194 \\ -19 & 2035 & -1515 \\ -19 & 3205 & 2633\end{array}$

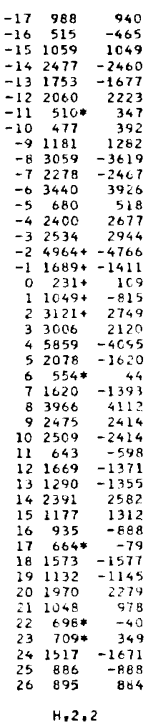

$\begin{array}{lll}-25 & 751 & -725 \\ -24 & -3394 & -325\end{array}$

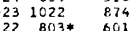

$\begin{array}{lll} & 21 \\ -20 & 777 * & -76 \\ -20 & 747 * & -239 \\ -10 & 6670 & -415\end{array}$

$\begin{array}{lrr}-19 & 7089 & -239 \\ -18 & 687 * & -415 \\ -17 & 803 & -415 \\ -17 & 803 & 824\end{array}$

$\begin{array}{rrr}-17 & 803 & 824 \\ -16 & 815 & 695 \\ -15 & 593 * & 429 \\ -14 & 565 & -125\end{array}$

$\begin{array}{lll}-14 & 565 * & -165 \\ -13 & 811 & -967 \\ -11 & \end{array}$

$\begin{array}{rlr}-12 & 811 & -967 \\ -11 & 613 * & -635 \\ -11 & 473 * & 122 \\ -10 & 742 & 712\end{array}$ $\begin{array}{rrr}-9 & 625 & 622 \\ -8 & 379 * & 148 \\ -7 & 428 & -407\end{array}$ $\begin{array}{lll}-7 & 428 & -407 \\ -6 & 864 & -850 \\ -5 & 286 * & -22 \\ -4 & 862 & -\end{array}$ $\begin{array}{lll}-4 & 772 & 601 \\ -3 & 826 & 776\end{array}$

$\begin{array}{rll}-2 & 996 & -938 \\ -1 & 591 & -542 \\ 0 & 249 & -240\end{array}$

$\begin{array}{lll}0 & 249 & -240 \\ 1 & 785 & -739\end{array}$

$\begin{array}{lll}2 & 1635 & 1543 \\ 3 & 451 & 424 \\ 4 & 543 & -520\end{array}$

$\begin{array}{lll}4 & 543 & -520 \\ 5 & 569 & 519 \\ 6 & 559 & -563 \\ 7 & 5510 & -51\end{array}$

$\begin{array}{lll}6 & 559 & -563 \\ 7 & 561 * & -191 \\ 7 & 562 & 481 \\ 8 & 502 & -916\end{array}$

$\begin{array}{rlr}8 & 562 & 481 \\ 9 & 792 & -816 \\ 10 & 567 * & 258\end{array}$

$\begin{array}{llr}11 & 871 & 854 \\ 12 & 887 & -793\end{array}$

$\begin{array}{rlr}13 & 595 * & 202 \\ 14 & 918 & 898 \\ 15 & 674 & -658 \\ 16 & 645 & -58\end{array}$

$\begin{array}{rlr}15 & 674 * & -658 \\ 16 & 624 * & -48 \\ 17 & 635 * & 369\end{array}$

$\begin{array}{llr}19 & 912 & -839 \\ 19 & 653 * & 450 \\ 20 & 656 & -687\end{array}$

$\begin{array}{lll}19 & 653 * & 450 \\ 20 & 666 & 667\end{array}$

$\begin{array}{llr}22 & 825 & -880 \\ 23 & 692 * & 133 \\ 24 & 700 & -651\end{array}$

$\mathrm{H.2}, 3$

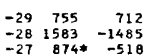

$\begin{array}{llll}-27 & 874 * & -1485 \\ -26 & 886 * & 418 \\ -476\end{array}$

$\begin{array}{lll}-25 & 872 * & -639 \\ -24 & 1474 & 1368 \\ -23 & \end{array}$

$\begin{array}{lll}-24 & 1474 & 1368 \\ -23 & 1163 & 1144 \\ -22 & 1670 & -1586 \\ -21 & 753 * & -350\end{array}$

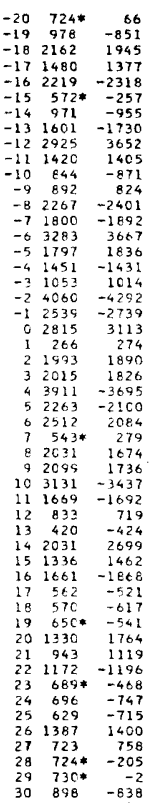

$\begin{array}{ccc}-21 & 887 & -576 \\ -20 & 1164 & 1110 \\ -19 & 1698 & -374\end{array}$ $\begin{array}{llll}-19 & 667 * & -374 \\ -18 & 1292 & 1247 \\ -17 & 1235 & 1093 \\ -16 & 2365 & -2263\end{array}$ $\begin{array}{lll}-17 & 1235 & 1093 \\ -16 & 2005 & -2203 \\ -15 & 2005 & -163\end{array}$ $\begin{array}{rrr}-15 & 858 & -853 \\ -15 & 858 \\ -14 & 119 & 1112 \\ -13 & 119 & -55\end{array}$ $\begin{array}{lll}-14 & 615 & -554 \\ -12 & 2148 & 2511 \\ -11 & 2087 & 2210 \\ -10 & 212 & -325\end{array}$ $\begin{array}{lll}-10 & 2712 & -3265 \\ -9 & 0126 & -16\end{array}$ $\begin{array}{rrr}-9 & 586 & -617 \\ -8 & 1216 & -1167 \\ -7 & 1055 & -376\end{array}$ $\begin{array}{rrr}-1 & 1655 & -1769 \\ -6 & 2820 & 3291 \\ -5 & 1595 & 1691 \\ -4 & 1995 & -292\end{array}$ $\begin{array}{rrr}-5 & 1595 & 1691 \\ -4 & 1555 & -2229 \\ -3 & 497 & -496\end{array}$

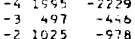

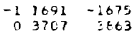
$\begin{array}{rrr}1 & 1942 & 1924 \\ 2 & 3467 & -1356 \\ 3 & 3458\end{array}$

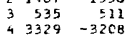

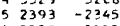
63309
71187
7
8 $\begin{array}{lll}8 & 386 & 363 \\ 9 & 837 & 787\end{array}$ $\begin{array}{lll}10 & 2498 & -2466 \\ 11 & 1535 & -1357 \\ 13 & 659 & 569\end{array}$ $\begin{array}{lll}12 & 1915 & 1934 \\ 13 & 658 & 693\end{array}$ $\begin{array}{lll}14 & 707 & 8=5 \\ 15 & 992 & 1067 \\ 17 & 109 & -326\end{array}$ $\begin{array}{lrr}16 & 2059 & -2526 \\ 17 & 191 & -1229 \\ 18 & 069 & -20\end{array}$ $\begin{array}{llr}19 & \text { OSE* } & -25 \\ 20 & 1152 & 1287\end{array}$

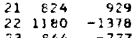
$\begin{array}{lll}25 & 69 \varepsilon^{*} & 193 \\ 25 & 701 * & -63 \\ 26 & 1005 & 1138\end{array}$

$$
\text { H.2.6 }
$$

$$
\mathrm{H}, 2.4
$$

$\begin{array}{llll}-23 & 1286 & 1156\end{array}$

$\begin{array}{lll}-20 & 848 & -843 \\ -19 & 006 * & -54\end{array}$

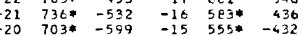

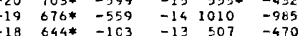

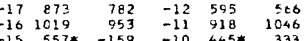
$\begin{array}{llllll}-15 & 557 * & -159 & -10 & 418 * & 345 \\ -14 & 751 & -690 & -9 & 427 * & -387 \\ -19 & 44 * & -925 & -9 & 04 & -79\end{array}$

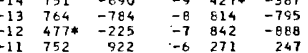

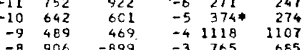
$\begin{array}{rrrrrr}-9 & 489 & 469 & -4 & 119 & 2164 \\ -8 & 906 & -899 & -3 & 765 & 685 \\ -7 & 1105 & -1130 & -2 & 1067 & -1070 \\ -6 & 3 & 205 & -1 & 300 & -202\end{array}$

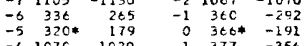

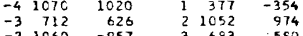

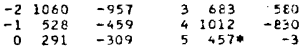
$\begin{array}{llllll}1 & 600 & -553 & 5 & 457 * & -3 \\ 2 & 1322 & 1159 & 7 & 484 * & 235 \\ 2 & 517 & -494\end{array}$

$\begin{array}{rrrrrr}3 & 533 & 471 & 8 & 676 & 629 \\ 4 & 383 & -391 & 9 & 569 * & 302 \\ 5 & 639 & 584 & 10 & 955 & -632 \\ 6 & 535 & -54 & 11 & 955 & -32\end{array}$

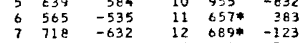
$\begin{array}{cccccc}8 & 1106 & 978 & 13 & 789 & -762 \\ 9 & 608 * & -54 & 14 & 784 & 682\end{array}$ $\begin{array}{llllll}10 & 470 & -549 & 15 & 640 * & -101 \\ 11 & 649 & 751 & 16 & 864 & -401\end{array}$ $\begin{array}{llllll}11 & 649 & 751 & 16 & 64 * & -401 \\ 12 & 441 & -457 & 17 & 813 & 882 \\ 13 & 435 & -395 & 18 & 68 * & -161\end{array}$ $\begin{array}{llllll}14 & 435 & -395 & 18 & 608 * & -161 \\ 15 & 993 & 1011 & 19 & 67 C * & -349\end{array}$ $\begin{array}{llllll}15 & 624 * & -514 & 20 & 678 * & 479 \\ 16 & 628 & -504 & 21 & 091 * & -506\end{array}$ $\begin{array}{rrrrrr}17 & 636 & 593 & 22 & 703 * & -203 \\ 18 & 642 * & -487 & 23 & 852 & 731\end{array}$ $\begin{array}{lllll}19 & 653 * & 6 & 6 \\ 20 & 657 & 608 & H .2 .7 \\ 21 & 816 & -625 & \end{array}$ $\mathrm{H}, 2,5$

$\begin{array}{lll}-26 & 1495 & 1275 \\ -25 & 635 * & 459 \\ -24 & 809 * & -743\end{array}$

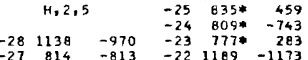

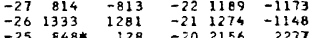
$\begin{array}{rrrrrr}-25 & 848 * & 128 & -20 & 2156 & 2277 \\ -24 & 114 * & 305 & -19 & 597 & 589 \\ -23 & 960 & 1072 & -18 & 642 * & 73\end{array}$ 
Table 7. Continued.

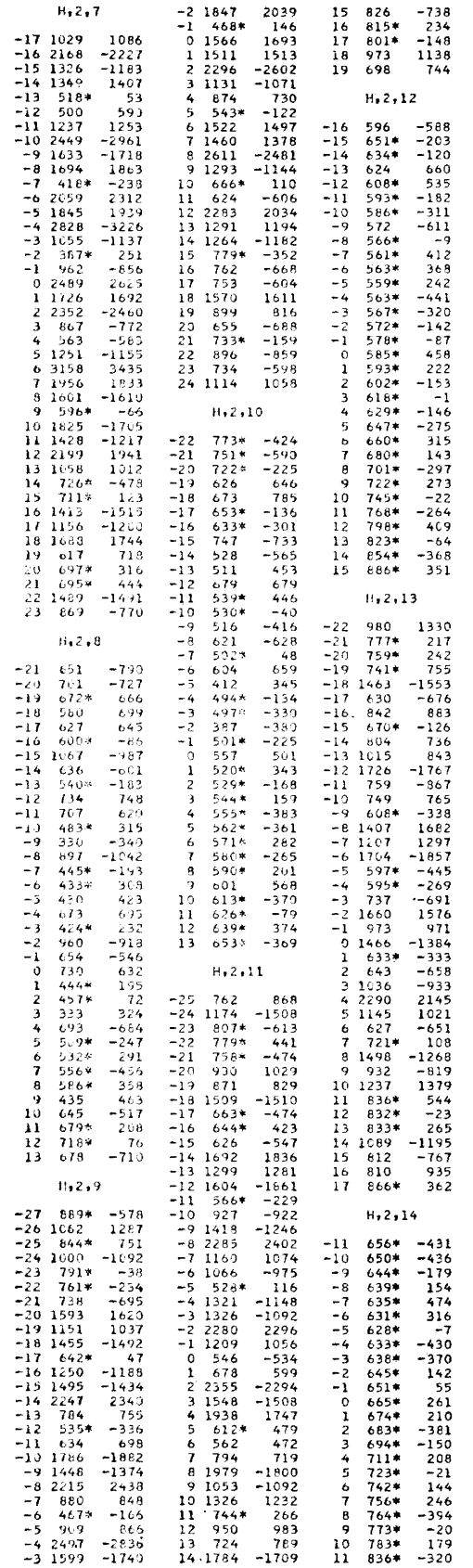

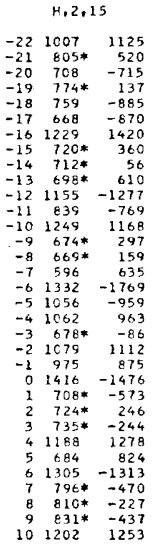

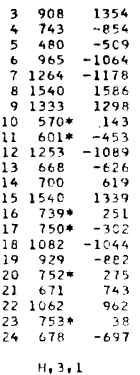

$\begin{array}{rrr}-28 & 712 & 871 \\ -27 & 975 & 940 \\ -225 & 9126 & -1121\end{array}$

$\begin{array}{lrr}-27 & 975 & -940 \\ -26 & 126 & -1121 \\ -25 & 129 & -1046 \\ -24 & 129 & -40\end{array}$

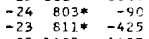

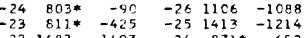

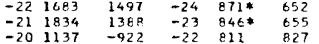
$\begin{array}{lll}-11 & 1300 & -622 \\ -18 & 757 & -662 \\ -134 & 734\end{array}$ $\begin{array}{lll}-18 & 757 & -734 \\ -17 & 1022 & -966 \\ -16 & 1887 & 1847\end{array}$

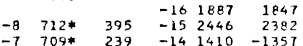

$\begin{array}{lll}-7 & 709 * & 239 \\ -6 & 706 * & 109 \\ -5 & 704 & -209 \\ -4 & 705 * & -406\end{array}$

$\begin{array}{lll}-5 & 704 \\ -5 & 704 * & -204 \\ -4 & 703 * & -401 \\ -3 & 702 & -194\end{array}$

$\begin{array}{lll}-11 & 2441 & -2735\end{array}$ $\begin{array}{ccc}-2 & 702 * & 344 \\ -1 & 703 * & 353\end{array}$

$\begin{array}{rrr}0 & 704 * & -100 \\ 1 & 707 * & 50 \\ 2 & 709 * & -272\end{array}$

$\begin{array}{lll}2 & 709 * & -272 \\ 3 & 713 * & -245 \\ 4 & 7121 & 325\end{array}$

$\begin{array}{lll}4 & 717 * & 323 \\ 5 & 721 * & 25 \\ 6 & 726 * & 76 \\ 7 & 72 * & \end{array}$

\begin{tabular}{llr}
$733 * * 242$ \\
$738 *$ & -327 \\
\hline $8,2,17$ &
\end{tabular}

$\mathrm{H}, 2,17$

$\begin{array}{rrr}-16 & 962 & 1078 \\ -15 & 693 & 685 \\ -14 & 695 & -15\end{array}$

$\begin{array}{lll}-15 & 693 & 689 \\ -14 & 685 & -915 \\ -13 & 70 * & 21 \\ -12 & 672 & -70 \\ -12 & 672 & -70\end{array}$

$\begin{array}{rrr}-11 & 667 & -745 \\ -10 & 1123 & 1323 \\ -12 & -12 & 1320\end{array}$

$\begin{array}{lll}-9 & 743 * & 498 \\ -8 & 740 * & -422 \\ -7 & 737 * & 149 \\ -6 & 740 & -675\end{array}$

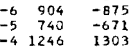

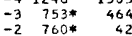

$\begin{array}{rrr}-1 & 768 * & 417 \\ 0 & 1298 & -1320 \\ 1 & 705 & -753\end{array}$

$\begin{array}{lll}2 & 714 & 841 \\ 3 & 812 * & 167 \\ 4 & 822 * & 489 \\ 5 & 824 & 431\end{array}$

$\begin{array}{rrr}4 & 822 * & 489 \\ 5 & 840 * & 531 \\ 6 & 1049 & -1122 \\ 7 & 864 * & -609\end{array}$

H,2,18

$\begin{array}{rrr}23 & 890 & 869 \\ 24 & 900 & -863 \\ 25 & 740 * & 337\end{array}$

$\begin{array}{llr}25 & 740 * & 337 \\ 26 & 746 * & -513 \\ 27 & 924 & -1112\end{array}$

$\begin{array}{rlr}-8 & 426 * & -26 \\ -7 & 913 & 967 \\ -6 & 2062 & -2464 \\ -5 & 2251 & -2585\end{array}$

$\begin{array}{rlr}-6 & 2062 & -2404 \\ -5 & 2251 & -2555 \\ -6 & 1151 & 256 \\ -4 & 1252 & 256\end{array}$

$\begin{array}{lll}-3 & 1252 & 2256 \\ -2 & 1299 \\ -2 & 6445 & 233\end{array}$

$\begin{array}{rrrr}-1 & 2385+ & 2031 \\ 0 & 3536+ & -3347\end{array}$

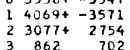

$\begin{array}{rrr}3 & 862 & 702 \\ 4 & 2612 & 1585 \\ 5 & 3668 & 3079 \\ 6 & 3320 & -2032\end{array}$

$\begin{array}{lll}5 & 3668 & 3079 \\ 6 & 3320 & -2932 \\ 7 & 1847 & -1724 \\ 9 & 1269 & -1039\end{array}$

$\begin{array}{rrr}8 & 625 & -639 \\ 9 & 1269 & -1039 \\ 9 & 1039 \\ 10 & 1877 & 1548 \\ 11 & 2629 & -2360\end{array}$

$\begin{array}{rrrr}10 & 1877 & 1548 \\ 11 & 2607 & 2382\end{array}$

$\begin{array}{lll}12 & 2628 & -2400 \\ 13 & 1459 & -1320 \\ 14 & 6744^{*} & 236 \\ 15 & 1892 & 139\end{array}$

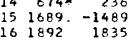

$\begin{array}{rlr}17 & 1965 & 1748 \\ 18 & 1721 & -1271 \\ 20 & 204 & -248\end{array}$

19 699* 66

$\begin{array}{lll}26 & 746 * & -513 \\ 27 & 924 & -112 \\ 28 & 930 & 11122\end{array}$

$\mathrm{H}, 3,2$

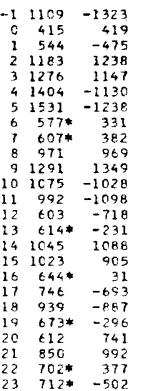

$\begin{array}{ccc}0 & 1560 & 1686 \\ 2 & 1314 & 1232 \\ 2 & 337 * & -144 \\ 3 & 010 & -130\end{array}$

$\begin{array}{rrr}3 & 010 & 550 \\ 4 & 1433 & -1229 \\ 5 & 53 & -128\end{array}$

$\begin{array}{rrr}5 & 1312 & -1182 \\ 6 & 586 & 51 \\ 7 & 645 & 668 \\ 7 & 649 & 45\end{array}$

$\begin{array}{lll}8 & 645 & 668 \\ 9 & 410 & 444 \\ 9 & 645 * & 457\end{array}$

$\begin{array}{lll}10 & 1251 & -1118 \\ 11 & 1285 & -1157\end{array}$

$\begin{array}{rrr}12 & 654 * & 97 \\ 13 & 642 * & 379\end{array}$

$\begin{array}{lll}13 & 642 * & 379 \\ 14 & 1135 & 1246 \\ 16 & 29 & -68\end{array}$

$\begin{array}{ccc}15 & 728 & 718 \\ 16 & 787 & -690 \\ 16 & 0628 & -54\end{array}$

$\begin{array}{lll}17 & 798 & -920 \\ 18 & 662 * & -547 \\ 20 & 629 & 258\end{array}$

$\begin{array}{lll}18 & 682 * & -547 \\ 19 & 667 * & 285 \\ 19 & 829 & 78\end{array}$

$\begin{array}{lll}20 & 829 & 768 \\ 21 & 6 E E * & 632 \\ 22 & 693 * & -127 \\ 2 & 602 & -12\end{array}$

$$
\mathrm{H}, 3,5
$$

$\begin{array}{lll}-26 & 808 & -1029 \\ -25 & 1055 & -894\end{array}$

$\begin{array}{rrr}-26 & 1025 & -1029 \\ -23 & 954 \\ -23 & 989 & 1030 \\ -22 & 105 & 130\end{array}$

$\begin{array}{rrrr} & 989 & 1030 \\ -22 & 775 * & 138 \\ -21 & 747 * & 185 \\ -20 & 247 & -184\end{array}$

$\begin{array}{lll}-20 & 1471 & -1442 \\ -19 & 1614 & -1713 \\ -10 & 1795 & 1291\end{array}$

$\begin{array}{cccc}-18 & 1351 & 1291 \\ -17 & 772 & 766 \\ -15 & 7428 & 1360\end{array}$

$\begin{array}{rrr}-16 & 912 & 963 \\ -15 & 1428 & 1340 \\ -14 & 1408 & -2128\end{array}$

$\begin{array}{lll}-20 & 1856 & -174 \\ -19 & 1463 & -1468 \\ -19 & 038 & -486\end{array}$

$\begin{array}{lll}-18 & 683 * & 46 \\ -27 & 545 & -487 \\ -15 & & \end{array}$

$\begin{array}{lll}-16 & 1681 & 1896 \\ -15 & 1815 & 1774 \\ -154 & 1400 & -1739\end{array}$

$\begin{array}{lll}-16 & 1601 & 1000 \\ -14 & 1400 & -1738 \\ -13 & 1325 & -1538 \\ -11 & 932 & -931\end{array}$

$\begin{array}{ccc}-11 & 493 * & -128 \\ -11 & 932 & -931 \\ -10 & 2091 & 2359\end{array}$

$\begin{array}{rrr}-1 & 2091 & 2359 \\ -9 & 2219 & 2524 \\ -8 & 1647 & -1754 \\ -7 & 542 & -54\end{array}$

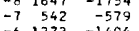

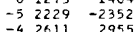

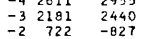

$\begin{array}{rrr}-1 & 595 & 555 \\ -1 & 595 & 555 \\ 0 & 1569 & -1894 \\ 1 & 569 & -295\end{array}$

$\begin{array}{rrr}1 & 2531 & -2673 \\ 2 & 2819 & 2952\end{array}$

$\begin{array}{rrr}3 & 2414 & 2314 \\ 4 & 960 & -769 \\ 5 & 1595 & -348\end{array}$

$\begin{array}{rrr}6 & 2910 & -2591 \\ 7 & 3322 & -2801 \\ 8 & 1990 & 1595\end{array}$

$\begin{array}{lll}8 & 199 \mathrm{C} & 1595 \\ 9 & 624 * & -0 \\ 0 & 675 & 750\end{array}$

$\begin{array}{rrr}11 & 1514 & 1609 \\ 12 & 1513 & -1771 \\ 13 & 1370 & -1571\end{array}$

$\begin{array}{lll}13 & 1370 & -1571 \\ 14 & 1241 & 1438\end{array}$

$\begin{array}{rrr}16 & 534 & 625 \\ 17 & 1505 & 1541 \\ 18 & 1751 & -1720\end{array}$

$\begin{array}{lll}18 & 1751 & -1720 \\ 19 & 1229 & -1000 \\ 20 & 205 & -560\end{array}$

$\begin{array}{rrr}20 & 605 & 530 \\ 21 & 839 & -767 \\ 22 & 852 & 868 \\ 23 & 150 & 253\end{array}$

$\begin{array}{rrrr}22 & 852 & 868 \\ 23 & 1180 & 1253 \\ 24 & 875 & \end{array}$

$$
\text { H.3.4 }
$$

$\begin{array}{rrr}-26 & 914 & 865 \\ -25 & 890 * & -636 \\ -24 & -20 & -20 \\ -23 & 9006 & 1027\end{array}$

$\begin{array}{ccc}-14 & 1908 & -211 \\ -13 & 1700 & -1728 \\ -12 & -12 & 742\end{array}$

$\begin{array}{llll} & 826 & 742 \\ -11 & 471 & 400\end{array}$

$\begin{array}{rrr}-9 & 1526 & 11612 \\ -8 & 1801 & -1967 \\ -7 & 1805 & -164\end{array}$

$\begin{array}{lll}-8 & 1855 & -1967 \\ -7 & 1859 & -1984\end{array}$

$\begin{array}{ccc}-6 & 625 & -650 \\ -5 & 601 & -604 \\ -4 & 295 & 250\end{array}$

$\begin{array}{lll}-4 & 2256 & -6474 \\ -3 & 2255 & 3253\end{array}$

$\begin{array}{lll}-4 & 2535 & 244 \\ -2 & 2390 & -2548 \\ -1 & 1058 & -1039 \\ 1 & 1790 & -134\end{array}$

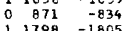

$\begin{array}{rrr}1 & 1798 & -1805 \\ 2 & 1907 & 1979 \\ 3 & 1876 & 1840 \\ 5 & 1515 & -1244\end{array}$

$\begin{array}{rrr}3 & 1876 & 1840 \\ 4 & 1512 & -1444 \\ 5 & 157 & -424 \\ 6 & 486 & -513\end{array}$

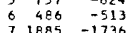

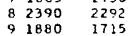

$\begin{array}{lll}10 & 944 & -\varepsilon 15\end{array}$

$\begin{array}{rrr}11 & 709 & 681 \\ 12 & 1401 & -1353 \\ 134 & 1300 & -168\end{array}$

$\begin{array}{rrr}13 & 1688 & -1842 \\ 14 & 1600 & 1601 \\ 15 & 033 & 0620\end{array}$

$\begin{array}{lll}15 & 933 & 837 \\ 16 & 662 * & -394 \\ 17 & 014 & -364\end{array}$

$\begin{array}{lll}117 & 814 & 847 \\ 18 & 1060 & -167 \\ 19 & 1133 & -1268 \\ 21 & 1262\end{array}$

$\begin{array}{lll}19 & 1133 & -1268 \\ 20 & 1002 & 1087\end{array}$

$\begin{array}{lll}21 & 694 * & 190 \\ 22 & 700 * & 137 \\ 24 & 1000 & -997\end{array}$

$\begin{array}{lll}23 & 1000 & 1063 \\ 24 & 1010 & -987\end{array}$

H, 3, 6

$\begin{array}{lll}-23 & 717 & 750 \\ -22 & 770 * & -339 \\ -21 & 65 & -731\end{array}$

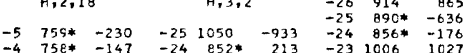

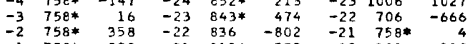

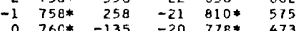

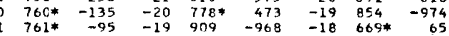

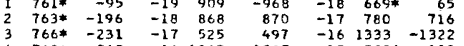

$\begin{array}{rrrrrrrrr}4 & 768 * & 242 & -16 & 1043 & -1085 & -15 & 580 * & -100 \\ 5 & 771 * & 77 & -15 & 871 & 840 & -14 & 834 & 811 \\ & & & -14 & 563 & -255 & -14 & 974 & -45\end{array}$

$$
\begin{array}{llll}
-14 & 583 * & -255 \\
-13 & 252 & -1358
\end{array}
$$

H,2,19

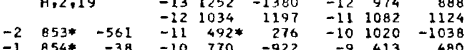

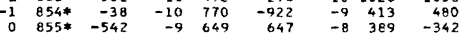

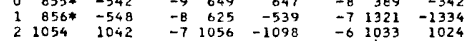

$\mathrm{H.3, \textrm {C }}$

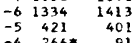

$\begin{array}{rrrrrr}1 & 777+ & -631 & -4 & 266 * & 91 \\ 2 & 1468+ & -3244 & -3189 & 1360 \\ 2 & 1468 & -2 & 1387 & -1664\end{array}$

Acta Chem. Scand. 23 (1969) No. 3 
Table $\%$. Continued.
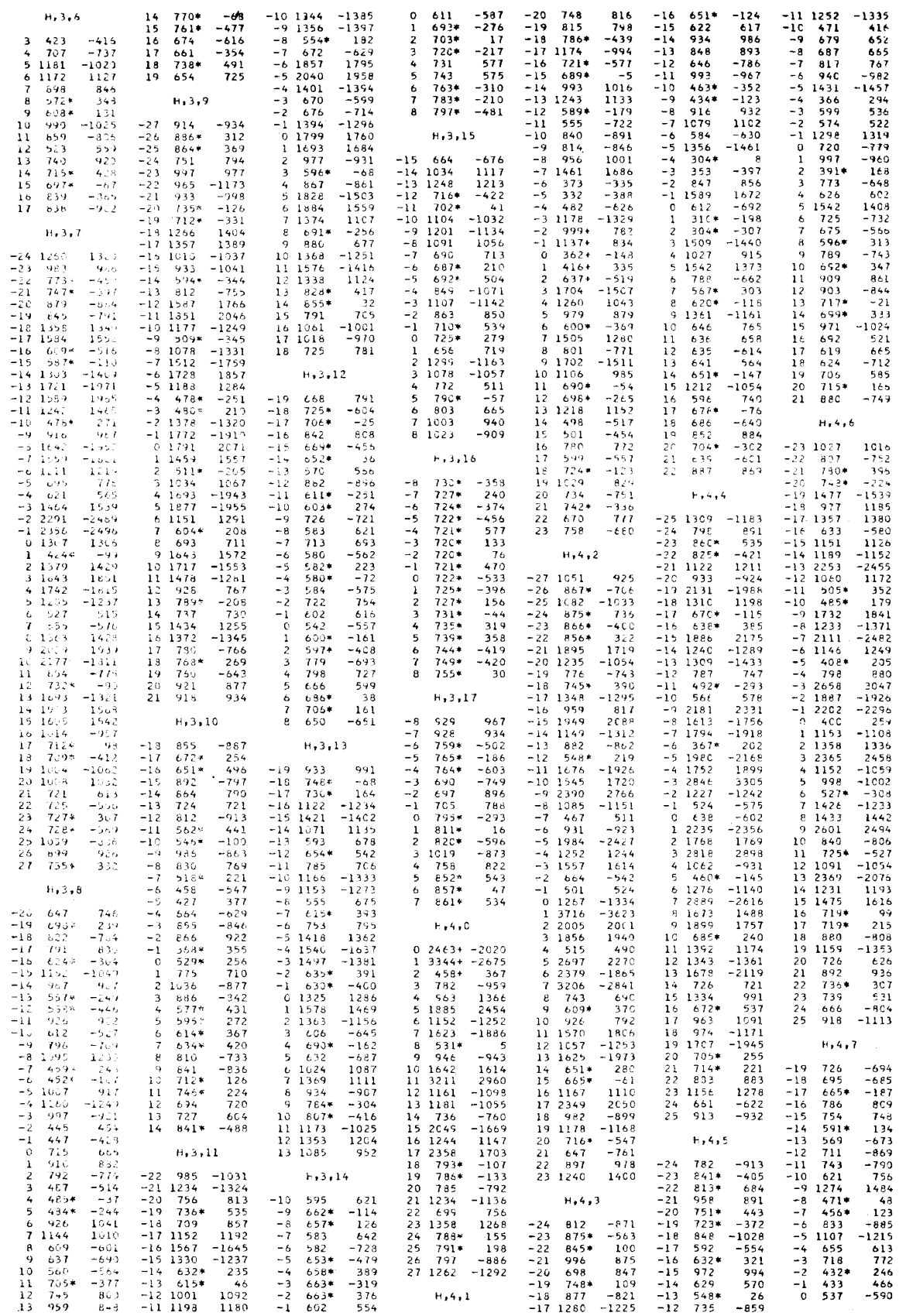

Acta Chem. Scand. 23 (1969) No. 3 
Table 7. Continued.

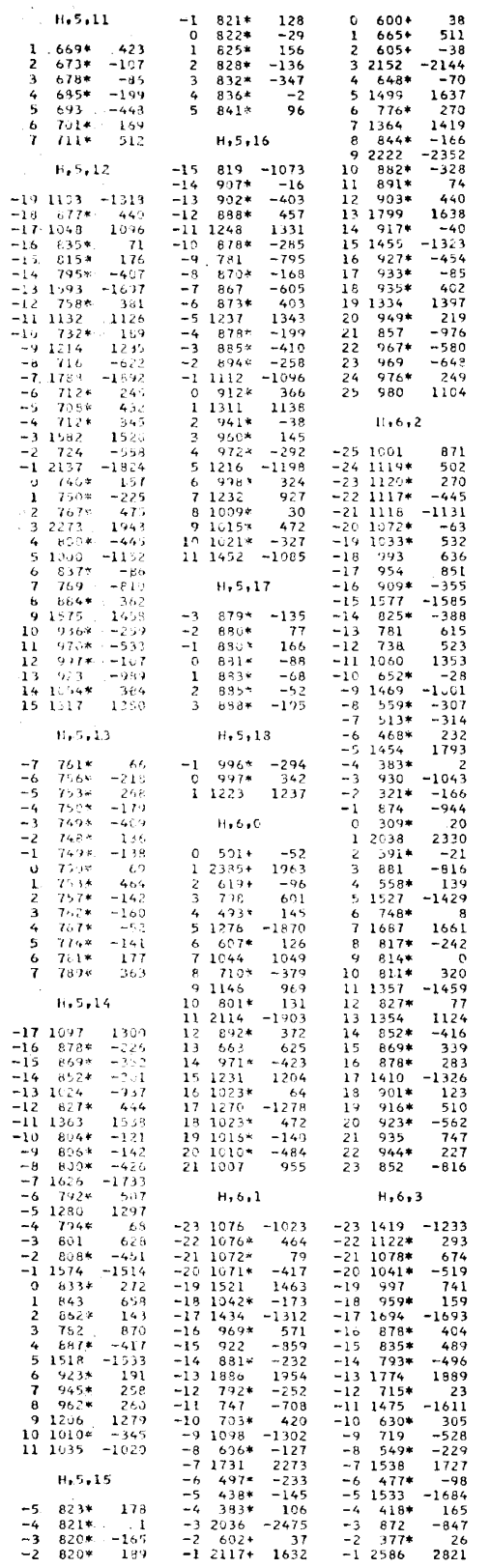

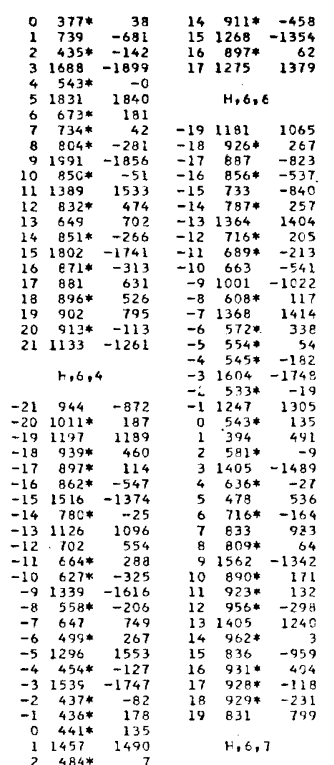

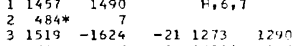

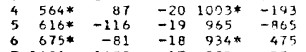

$\begin{array}{cccccc}6 & 675 * & -81 & -18 & 934 * & 475 \\ 7 & 1680 & 1498 & -17 & 803 & -976 \\ 8 & 812 * & -47 & -16 & 866 * & -156\end{array}$

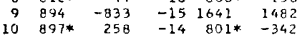

$\begin{array}{llllll}10 & 897 * & 258 & -14 & 801 * & -342 \\ 11 & 1106 & -975 & -13 & 766 * & -487\end{array}$

$\begin{array}{llllll}12 & 882 * & -286 & -12 & 739 * & 366 \\ 13 & 1479 * & 1342 & -11 & 1353 & -1361 \\ 14 & 870 * & -256 & -10 & 689 * & 30\end{array}$

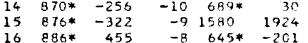

$\begin{array}{llr}16 & 886 * & 455 \\ 17 & 894 & -744 \\ 18 & 900 * & -23 \\ 19 & 110 & -978\end{array}$

$\begin{array}{ccc}18 & 900 * & -23 \\ 19 & 1110 & 977\end{array}$

$4,6,5$

$\begin{array}{lll}-21 & 1275 & 1300 \\ -20 & 16 C 4 * & -471 \\ -19 & 693 * & 276\end{array}$

$\begin{array}{ccc}-20 & 1664 * & -471 \\ -19 & 963 * & 276 \\ -19 & 924 & 418\end{array}$

$\begin{array}{rrr}-18 & 928 * & 418 \\ -17 & 1091 & -1335 \\ -16 & 854 * & 102 \\ -15 & 1363 & 1156 \\ -14 & 77 \% & -471\end{array}$

$\begin{array}{rrr}-16 & 854 * & 102 \\ -15 & 1363 & 1156 \\ -14 & 776 * & -471 \\ -13 & 738 * & 339 \\ -12 & 730 * & 215\end{array}$

$\begin{array}{rrr}-12 & 702 * & 215 \\ -11 & 1887 & -2163 \\ -10 & 638 * & 178\end{array}$

$\begin{array}{rrrr}-10 & 638 * & 178 \\ -9 & 1019 & 1022 \\ -8 & 579 * & -231 \\ -7 & 139 *\end{array}$

$\begin{array}{lrr}-8 & 579 * & -231 \\ -7 & 1383 & 1519 \\ -6 & 532 * & 61 \\ -5 & 1593 & -1930 \\ -6 & 590 * & 127\end{array}$

$\begin{array}{rrr}-5 & 532 * \\ -5 & 1593 & -1930 \\ -4 & 500 * & 127\end{array}$

$\begin{array}{lll}-4 & 500 * & 127 \\ -3 & 490 * & 328 \\ -1 & 4511 & -63\end{array}$

$\begin{array}{rrrr}-2 & 484 * & -63 \\ -1 & 1511 & 1912 \\ 0 & 492 * & -57\end{array}$

$\begin{array}{cccc}1 & 1788 & -1973 \\ 2 & 530 * & -117 \\ 3 & 688 & -630 \\ 5 & 680 * & -630 \\ 5 & 2016 & 2161\end{array}$

$\begin{array}{rrrrr}-630 & -15 & 850 * & -493 \\ 205 & -14\end{array}$

$\begin{array}{rrrrrr}4 & 600 * & 154 & -14 & 823 * & 483 \\ 5 & 2016 & 2161 & -13 & 1227 & 1337\end{array}$

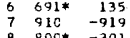

$\begin{array}{rlll}8 & 800 * & -301 \\ 9 & 946 & -920 \\ 10 & 918 * & -929 \\ 11 & 918 & 97\end{array}$

$\begin{array}{rrr}10 & 946 * & -929 \\ 11 & 181 \mathrm{C} & 97 \\ 12 & 953 * & 1707 \\ 12 & 927 & -620\end{array}$

$\begin{array}{llll}-5 & 1859 & -2184 \\ - & -180\end{array}$

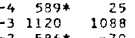

$\begin{array}{rrr}-2 & 586 * & -70 \\ -1 & 985 & 948 \\ 0 & 599 * & 940 \\ 1 & 598 * & -110\end{array}$

$\begin{array}{lrr}0 & 599 * & -10 \\ 1 & 1786 & -1771 \\ 2 & 626 * & -18 \\ 3 & 1020 & -185\end{array}$

$\begin{array}{rrr}3 & 1028 & 965 \\ 4 & 680 * & 183 \\ 5 & 1371 & 1209 \\ 6 & 1760 & -1200\end{array}$

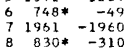

$\begin{array}{rrr}8 & 830 * & -310 \\ 9 & 874 * & -57 \\ 10 & 925 * & 323 \\ 11 & 1639 & 1566\end{array}$

$\begin{array}{rrr}11 & 1539 & 1566 \\ 12 & 1020 * & 78 \\ 13 & 1064 & -1056\end{array}$

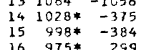

$\begin{array}{ccc}16 & 975 * & 299 \\ 17 & 1351 & 1337\end{array}$

$$
H, 6, \varepsilon
$$

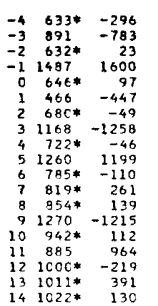

14
$15022 *$
1533

$$
r, t, 9
$$

$\begin{array}{lll}-19 & 1214 & -1281 \\ -18 & 960 * & 389 \\ -12 & 925 * & 260\end{array}$

$\begin{array}{rrr}-19 & 1214 & -128 \\ -17 & 925 * & 260 \\ -16 & 898 * & -317 \\ -15 & 1797 & 157\end{array}$

$\begin{array}{lllr}-14 & \varepsilon 38 * & -55 \\ -13 & 1358 & -1155 \\ -12 & 388 & 352\end{array}$

$\begin{array}{llll}-12 & 783 * & 362 \\ -11 & 680 & -659 \\ -10 & 735 & -178\end{array}$

$\begin{array}{llll}-9 & 1392 & -178 \\ -8 & 707 * & -151 \\ -151 & -1 & 301 & -850\end{array}$

$\begin{array}{rrr}-7 & 851 & -850 \\ -6 & 682 * & 127\end{array}$

$\begin{array}{ccc}-4 & 676 * & 39 \\ -3 & 1964 & 1932 \\ -3 & 698\end{array}$

$\begin{array}{lll}-2 & 684 * & -15 \\ -1 & 690 * & -342 \\ -1 & 69.4 & -1562\end{array}$

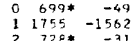

$\begin{array}{lll}2 & 728 * & -31 \\ 3 & 1548 & 1366 \\ 4 & 770 * & 154 \\ 6 & 772 & 153\end{array}$

$\begin{array}{cccc}4 & 77 C * & 154 \\ 5 & 794 * & 173 \\ 6 & 172 * & -138\end{array}$

$\begin{array}{lll}6 & 826 * & -178 \\ 7 & 1759 \\ 8 & 859 * & -1592 \\ 8 & 8896 & -36\end{array}$

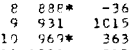

$\begin{array}{lll}11 & 1008 & 913 \\ 12 & 1055 * & -198\end{array}$

$13 \quad 1552-1330$

$H, 6,10$

$\begin{array}{rrr}-17 & 944 & -1058 \\ -16 & 911 * & -60 \\ -15 & 91 \% & 693\end{array}$

$\begin{array}{ccc}-16 & 911 * & -650 \\ -15 & 911 * & -60 \\ -15 & 791 & 807 \\ -19 & 19 & 30\end{array}$

$\begin{array}{llll}-14 & 8 \in 2 * & 633 \\ -13 & 835 * & 370 \\ -12 & 017 * & -228\end{array}$

$\begin{array}{lll}-12 & 817 * & -228 \\ -11 & 1325 & -1400 \\ -145 & 1754 & -277\end{array}$

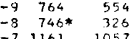

$\begin{array}{lll}-8 & 746 * & 326 \\ -7 & 1161 & 1057 \\ -t & 725 * & -79 \\ -5 & 1428 & -250\end{array}$

$\begin{array}{llll}-5 & 1488 & -1250 \\ -4 & 726 * & -218 \\ -3 & 730 * & -15\end{array}$

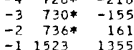

$\begin{array}{rrrr}-1 & 1523 & 1355 \\ 0 & 752 * & 11\end{array}$

$\begin{array}{rrr}0 & 752 * & 11 \\ 1 & 1276 & -1114 \\ 2 & 781 * & -16\end{array}$

$\begin{array}{llr}2 & 781 * & -76 \\ 3 & 802 * & -337 \\ 4 & 817 * & 32 \\ 5 & 168 * & -42\end{array}$

$\begin{array}{rrr}4 & 817 * & 32 \\ 5 & 1666 & 1412 \\ 6 & 866 * & -21 \\ 7 & 860 \% & -573\end{array}$

$\begin{array}{lll}6 & 866 * & -21 \\ 7 & 900 & -573 \\ 7 & 928 * & 105 \\ 9 & 982 & -105\end{array}$

$\begin{array}{ccc}6 & 928 * & 105 \\ 9 & 881 & -812 \\ 10 & 1001 * & -47 \\ 10 & 028 & 100\end{array}$

$$
H, t, 11
$$

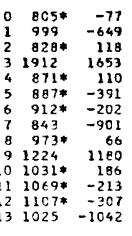

$H, \epsilon, 12$

$\begin{array}{lll}-15 & 834 \\ -14 & 873 * & 1066 \\ -13 & 059 & -122\end{array}$

$\begin{array}{lll}-13 & 870 * & -122 \\ -12 & 859 * & -484 \\ -11 & 757 & -939\end{array}$

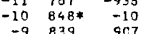

$\begin{array}{lll}-9 & 839 & 967 \\ -9 & 831 * & 333\end{array}$

$\begin{array}{llr}-7 & 824 * & 378 \\ -6 & 819 * & -214 \\ -5 & 1679 & -146\end{array}$

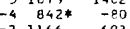

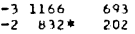

$\begin{array}{llll}-2 & 832 * & 202 \\ -1 & 754 & 817 \\ 0 & 957 * & -93\end{array}$

$\begin{array}{rrr}0 & 851 * & -93 \\ 1 & 1372 & -9196 \\ 2 & 881 * & -59 \\ 3 & 9120 & 340\end{array}$

3 902* 340

$\begin{array}{rrr}4 & 916 * & 78 \\ 5 & 840^{\circ} & 850 \\ 6 & 969^{2} & 37\end{array}$

$\begin{array}{lll}6 & 965 * & 37 \\ 7 & 883 & -923 \\ 9 & 1025 & -22\end{array}$

$\begin{array}{lll}8 & 1017 * & 21 \\ 9 & 1045 * & -272\end{array}$

$11987 \quad 2611$

$\begin{array}{llr}12 & 114 C * & 15 \\ 13 & 1170 * & -536\end{array}$

$\mathrm{H}, 6,13$

$\begin{array}{rrr}-13 & 824 \\ -12 & 909 * & -856 \\ -11 & 1095 & 83\end{array}$

$\begin{array}{rrrr}-12 & 909 * & 83 \\ -11 & 1095 & 137 \\ -10 & 883 * & -152 \\ -2 & 895 * & \end{array}$

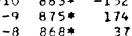

$\begin{array}{rrrr}-7 & 1448 & -1567 \\ -5 & 866 * & 55 \\ -5 & 060 & 59\end{array}$

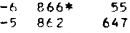

$\begin{array}{lll}-4 & 8<8 * & -39 \\ -3 & 875 & 839 \\ -2 & 872 & -32\end{array}$

$\begin{array}{lll}-2 & 882 * & -22 \\ -1 & 125 & 0\end{array}$

$\begin{array}{rrr}1 & 1259 & -222 \\ 0 & 901 * & 20 \\ 1 & 920 \% & 359 \\ 2 & 020 & 125\end{array}$

$\begin{array}{lll}1 & 920 * & 359 \\ 3 & 932 * & 125\end{array}$

$\begin{array}{cccc}3 & 1165 & 1128 \\ 4 & 968 * & -45 \\ 6 & 1208 & -458\end{array}$

$\begin{array}{rlr}4 & 968 * & -45 \\ 5 & 1208 & -11 c 8 \\ 7 & 1208 * & -220\end{array}$

$\begin{array}{lll}4 & 1008 * & -220 \\ 7 & 103 * * & -168 \\ 8 & 1059 * & 144 \\ 8 & \end{array}$

$$
H, t, 14
$$

$\begin{array}{llll}-5 & 813 & -787 \\ -4 & 916 * & 45 \\ -3 & 923 & -93\end{array}$

$\begin{array}{ccc}-4 & 916 * & 45 \\ -3 & 923 & 1573 \\ -2 & 923 & 0\end{array}$

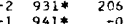

$\begin{array}{lll}1 & 941 * & -0 \\ 0 & 959 * & -110 \\ 1 & 868 & -965\end{array}$

\begin{tabular}{lll}
1 & 868 & -965 \\
2 & $982 *$ & -39 \\
3 & 995 & 572 \\
\hline & $995 *$ & 56
\end{tabular}

$\begin{array}{lll}4 & 1018 * \\ 5 & 1033 * & 61 \\ 6 & 411\end{array}$

$\begin{array}{lll}6 & 1056 * & 32 \\ 7 & 963 & -873\end{array}$

$$
\text { H.6.15 }
$$

$\begin{array}{rrr}-5 & 954 & 1138 \\ -4 & 961 * & -17 \\ -3 & 969 * & 81\end{array}$

$\begin{array}{rlr}-3 & 969 * & 81 \\ -2 & 978 * & -18 \\ -1 & 987 & -1014 \\ -1 & 998 & -12\end{array}$

$\begin{array}{rrr}-1 & 987 & -1014 \\ 0 & 989 * & -20 \\ 1 & 992 & 759\end{array}$

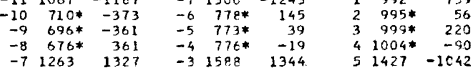

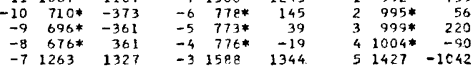

Acta Chem. Scand. 23 (1969) No. 3 
Table \%. Continued.

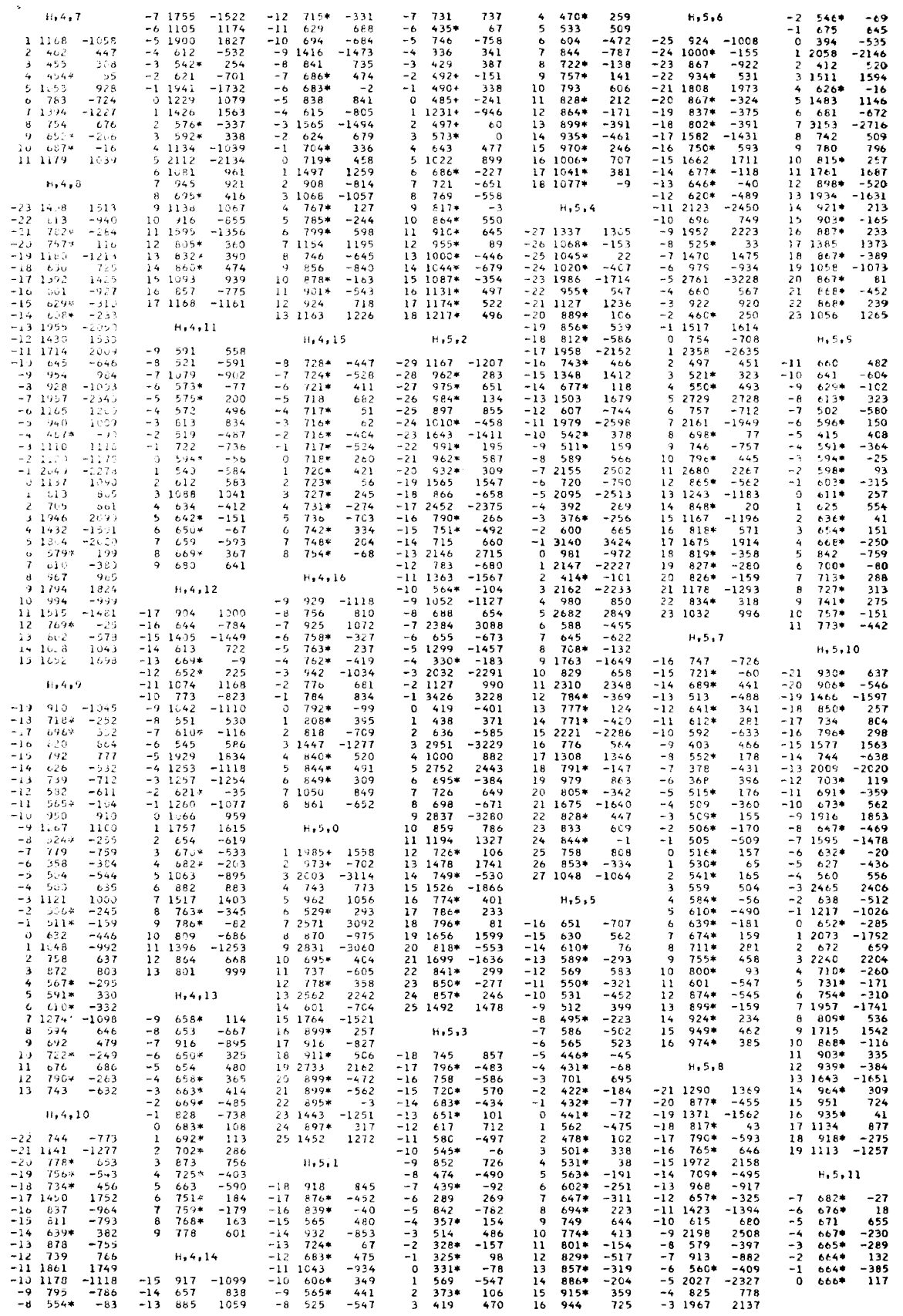

Acta Chem. Scand. 23 (1969) No. 3 
as peaks of average heights 47 and 17 electrons $/ \AA^{3}$, respectively. The highest electron density of the background was about 10 electrons $/ \AA^{3}$ but peaks of the height 30 electrons $/ \AA^{3}$ were found close to the uranium atoms.

All atoms were now included for a new series of least squares refinement. The scattering factors were those given by Cromer and Waber ${ }^{7}$ for uranium and chlorine and by Berghuis et al. ${ }^{8}$ for oxygen. For the uranium and chlorine atoms the real part of the dispersion correction was introduced. ${ }^{9}$ Hughes' weighting scheme was used. ${ }^{10}$ In addition to the positional parameters, individual scale factors for the seven layers and isotropic temperature factors for all atoms were refined. The $R$ factor was lowered from 0.257 to 0.095 in four cycles.

In the difference Fourier map mentioned above, indications of anisotropic vibrations of the uranium and chlorine atoms were observed. Thus a refinement including anisotropic temperature factors for these atoms was carried out. At this stage an overall scale factor was introduced because of the risk of degeneracy between interlayer scale factors and anisotropic temperature factors expressed by, e.g., Lingafelter and Donohue. ${ }^{11}$ This refinement gave a final $R$ factor of 0.091 after three cycles. In the last cycle all shifts in the parameters were less than $0.1 \%$ of the calculated standard deviations. The final parameters and temperature factors based on the MoKa data are given in Table 6 and observed and calculated structure factors obtained in the last cycle of the refinement are listed in Table 7.

A new difference Fourier map indicated that the accuracy of the experimental data was not sufficient to make an anisotropic refinement of the oxygen positions meaningful. Nevertheless an attempt was made to include anisotropic temperature factors for all atoms in a refinement which, however, resulted in some of the thermal parameters of five of the oxygen atoms being negative. In another refinement the imaginary part of the dispersion correction for the uranium and chlorine atoms ${ }^{9}$ was included. No significant differences in the parameters and no change in the $R$ factor were obtained.

To check the results of the least squares refinement and to confirm that no other atoms except hydrogen atoms were present in the unit cell, a final three-dimensional electron density map and a three-dimensional difference map were calculated using the parameters obtained in the last cycle of the least squares refinement and with all observed reflections included. All peaks in the difference map were less than 6 electrons $/ \AA^{3}$ (the average height of an oxygen peak was 20 electrons $/ \AA^{3}$ ) except for some spurious peaks close to the uranium atoms which had a height of about 10 electrons $/ \AA^{3}$. These are most probably due to the uncertainty in the absorption correction.

The $\mathrm{CuK} \alpha$ data were refined in the same way as described above for the MoK$K$ data. The final $R$ factor was 0.099 . The differences in the positional parameters between the two sets of data were for all values within three times the standard deviations with an average root mean square deviation of $0.05 \AA$ for the oxygen atoms. However, the temperature factors calculated from the $\mathrm{Cu} K \alpha$ data were about three times larger than those from the MoK$\alpha$ data. This is probably due to a decomposition of the crystal during the investigation and the significance of the $B$ values given in Table 6 may, therefore, be doubtful.

Acta Chem. Scand. 23 (1969) No. 3 

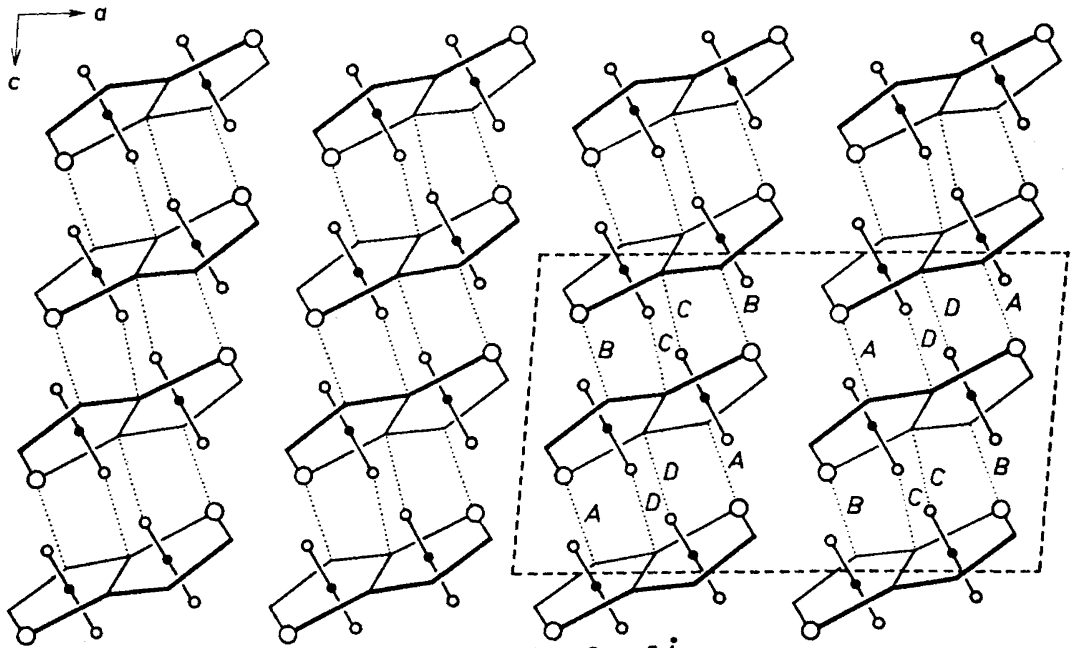

Fig. 1. Projection of the structure along the $b$ axis. Uranium atoms are indicated by filled circles, uranyl oxygen atoms by small and chlorine atoms by large open circles. Other atoms are not marked. Those edges which are at a higher level are drawn as thick lines. The unit cell is indicated by dashed and possible hydrogen bonds by dotted lines. The letters refer to hydrogen bonds discussed in the text.
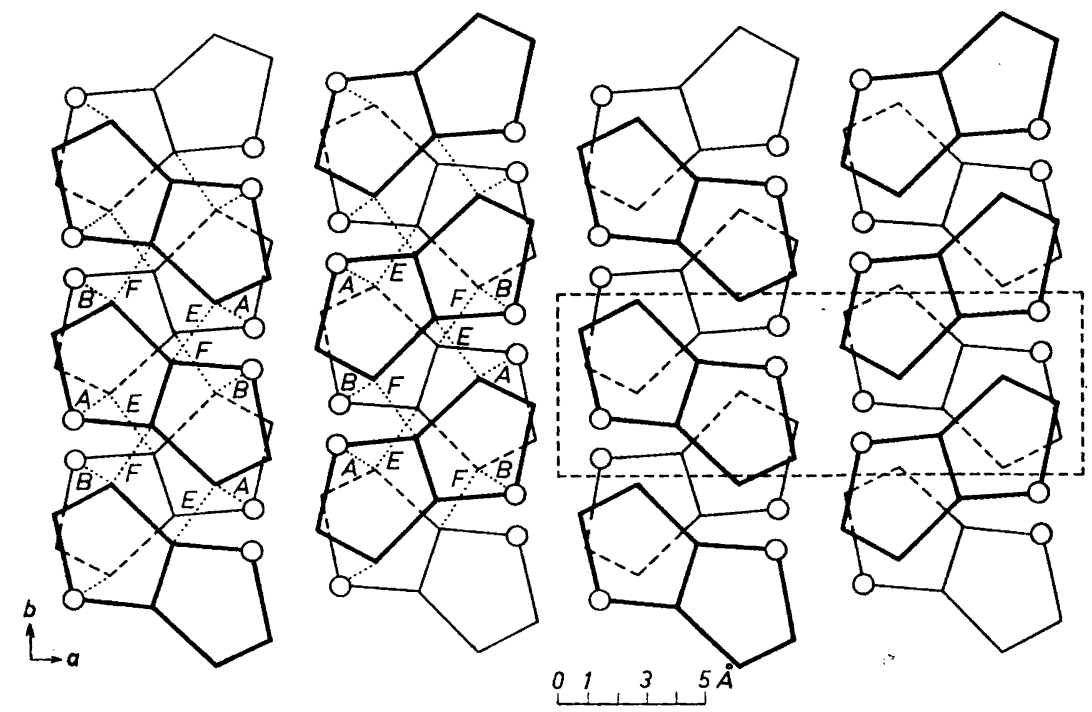

Fig. 2. Projection of the structure along the $c$ axis. Designations as before except that the uranyl groups are left out for clarity. 


\section{DESCRIPTION OF THE STRUCTURE}

Projections of the structure along the two shortest axes of the unit cell, the $b$ and $c$ axes, are shown in Figs. 1 and 2. The structure can be viewed as being built up from dimers of uranium atoms bonded by double oxygen bridges. The $U(1)-U(2)$ distance within the dimer is $3.94 \AA$. The shortest distances between uranium atoms belonging to different dimers are 5.61 $\AA$ in the $a$ axis direction, $5.62 \AA$ in the $b$ axis direction, and 5.50 and $5.54 \AA$ in the $c$ axis direction.

The structure of one dimer is shown in Fig. 3. Some important bond lengths and angles within the dimer are given in Table 8. Each of the two uranium atoms is surrounded by one chlorine and six oxygens at the vertices of a pentagonal bipyramid. Since two oxygens are shared between the uraniums

Table 8. Some important interatomic distances and angles. Standard deviations are given in parentheses.

\begin{tabular}{|c|c|c|c|}
\hline & & & \\
\hline $\mathrm{U}(1)-\mathrm{U}(2)$ & $3.944(1)$ & & \\
\hline$-\mathrm{Cl}(1)$ & $2.754(8)$ & $\mathrm{U}(2)-\mathrm{Cl}(2)$ & $2.748(8)$ \\
\hline$-O(1)$ & $2.321(23)$ & $-\mathrm{O}(1)$ & $2.391(21)$ \\
\hline$-O(2)$ & $2.396(19)$ & $-\mathrm{O}(2)$ & $2.349(21)$ \\
\hline$-O(3)$ & $1.821(21)$ & $-\mathrm{O}(5)$ & $1.812(20)$ \\
\hline$-O(4)$ & $1.752(15)$ & $-O(6)$ & $1.760(20)$ \\
\hline$-O(7)$ & $2.366(21)$ & $-\mathrm{O}(9)$ & $2.406(20)$ \\
\hline$-\mathrm{O}(8)$ & $2.533(21)$ & $-O(10)$ & $2.462(32)$ \\
\hline $\mathrm{Cl}(1)-\mathrm{O}(1)$ & $3.143(22)$ & $\mathrm{Cl}(2)-\mathrm{O}(2)$ & $3.134(21)$ \\
\hline$-\mathrm{O}(3)$ & $3.379(22)$ & $-O(5)$ & $3.271(21)$ \\
\hline$-\mathrm{O}(4)$ & $3.228(17)$ & $-O(6)$ & $3.334(22)$ \\
\hline$-O(8)$ & $3.179(23)$ & $-O(10)$ & $3.108(33)$ \\
\hline$O(1)-O(2)$ & $2.61(3)$ & & \\
\hline$-\mathrm{O}(3)$ & $2.98(3)$ & $\mathrm{O}(2)-\mathrm{O}(5)$ & $2.94(3)$ \\
\hline$-O(4)$ & $2.87(3)$ & $-O(6)$ & $2.98(3)$ \\
\hline$-O(9)$ & $2.90(3)$ & $-O(7)$ & $2.93(3)$ \\
\hline $\mathrm{O}(7)-\mathrm{O}(3)$ & $2.95(3)$ & $\mathrm{O}(9)-\mathrm{O}(5)$ & $3.06(3)$ \\
\hline$-O(4)$ & $2.97(3)$ & $-\mathrm{O}(6)$ & $2.89(3)$ \\
\hline$-\mathrm{O}(8)$ & $2.71(3)$ & $-O(10)$ & $2.81(4)$ \\
\hline $\mathrm{O}(8)-\mathrm{O}(3)$ & $3.07(3)$ & $\mathrm{O}(10)-\mathrm{O}(5)$ & $3.10(4)$ \\
\hline$-O(4)$ & $3.16(3)$ & $-\mathrm{O}(6)$ & $2.99(4)$ \\
\hline & Angle $\left({ }^{\circ}\right)$ & & Angle $\left({ }^{\circ}\right)$ \\
\hline $\mathrm{Cl}(1)-\mathrm{U}(1)-\mathrm{O}(1)$ & $76.0(6)$ & $\mathrm{Cl}(2)-\mathrm{U}(2)-\mathrm{O}(2)$ & $75.4(5)$ \\
\hline $\mathrm{O}(1)-\quad-\mathrm{O}(2)$ & $67.2(7)$ & $O(2)-\quad-O(1)$ & $66.8(7)$ \\
\hline $\mathrm{O}(2)-$ & $76.0(7)$ & $-O(9)$ & $74.3(7)$ \\
\hline$O(7)-$ & $67.1(7)$ & $-O(10)$ & $70.5(9)$ \\
\hline $\mathrm{O}(8)-$ & $73.8(5)$ & $\mathrm{O}(10)-$ & $73.0(8)$ \\
\hline $\mathrm{O}(3)-$ & $178.3(8)$ & $\mathrm{O}(5)-$ & $178.0(9)$ \\
\hline $\begin{array}{c}U(1)-O(1)-U(2) \\
-O(2)-\end{array}$ & $\begin{array}{l}113.6(9) \\
112.4(8)\end{array}$ & & \\
\hline
\end{tabular}

Acta Chem. Scand. 23 (1969) No. 3 

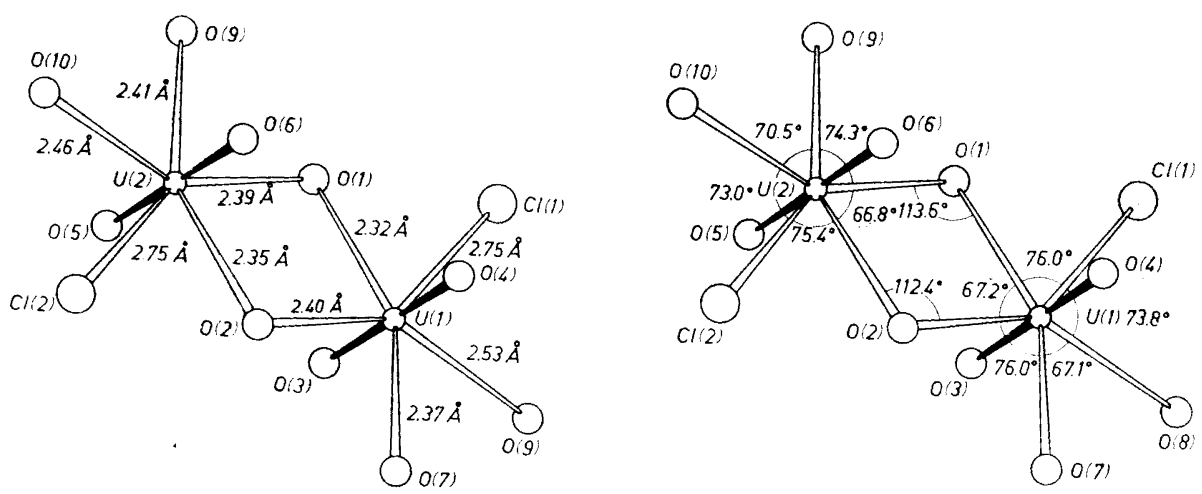

Fig. 3. The discrete group $\left[\left(\mathrm{UO}_{2}\right)_{2}(\mathrm{OH})_{2} \mathrm{Cl}_{2}\left(\mathrm{H}_{2} \mathrm{O}\right)_{4}\right]$ viewed along the $b$ axis. $\mathrm{O}(1)$ and $\mathrm{O}(2)$ form a double oxygen bridge. $\mathrm{O}(3), \mathrm{O}(4), \mathrm{O}(5)$, and $\mathrm{O}(6)$ are the uranyl oxygen atoms whose bonds to the uranium atoms are filled in the figures. $\mathrm{O}(7), \mathrm{O}(8), \mathrm{O}(9)$, and $\mathrm{O}(10)$ are water oxygen atoms.

the dimer contains in total two chlorine and ten oxygen atoms. Four of these oxygens, $\mathrm{O}(3), \mathrm{O}(4), \mathrm{O}(5)$, and $\mathrm{O}(6)$, are more strongly bonded to the uranium atoms than the others. The distances vary between 1.75 and $1.82 \AA$ with an average value of $1.79 \AA$. These oxygens form together with the uranium atoms two uranyl groups. The angles $\mathrm{O}(3)-\mathrm{U}(1)-\mathrm{O}(4)$ and $\mathrm{O}(5)-\mathrm{U}(2)-\mathrm{O}(6)$ are both $178^{\circ}$ which is not significantly different from $180^{\circ}$, and the uranyl groups are thus linear. They also lie in the same plane. The deviations of the six atoms from the mean plane $0.267 x+0.894 y-0.531 z-0.461=0$ obtained by the method of least squares are not significant (Fig. 4a).

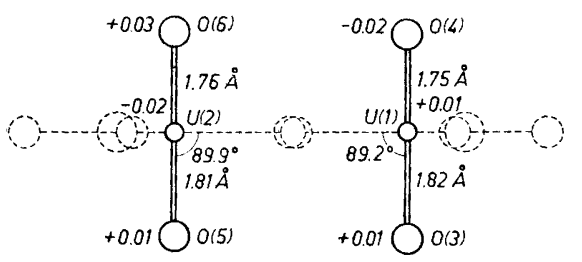

Fig. 4a. Plane through the linear uranyl groups. The dashed lines and circles indicate the rings. The figures give the deviations in $\AA$ (with sign) from the best mean plane obtained by the method of least squares.

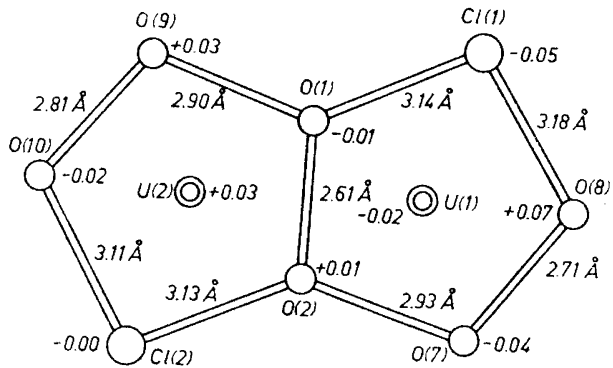

Fig. 4b. Plane through the two coplanar five-membered rings and the uranium atoms. The figures give the deviations in $\AA$ (with sign) from the least squares plane. 
S T R U C T URE OF $\left[\left(\mathrm{UO}_{2}\right)_{2}(\mathrm{OH})_{2} \mathrm{Cl}_{2}\left(\mathrm{H}_{2} \mathrm{O}\right)_{4}\right]$

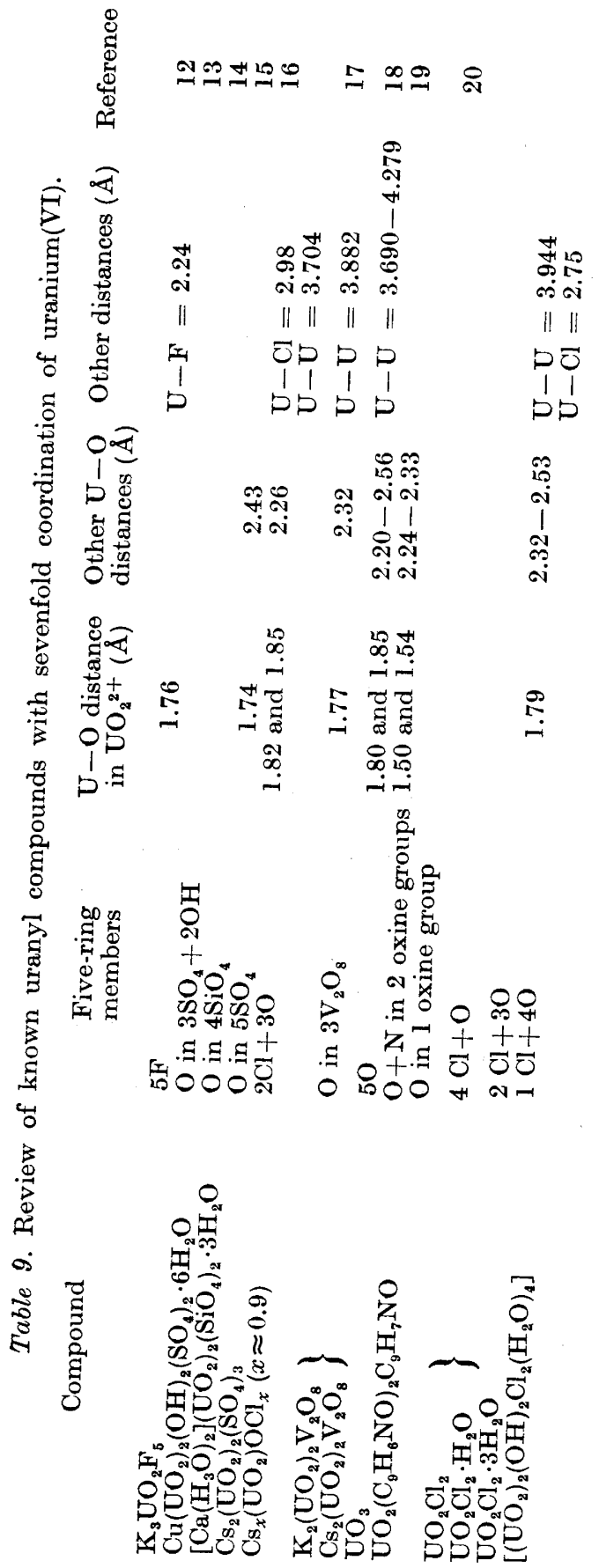

Acta Chem. Scand. 23 (1969) No. 3 
The $\mathrm{U}-\mathrm{O}$ distances within the bridge formed by $\mathrm{O}(\mathrm{l})$ and $\mathrm{O}(2)$ are 2.32 , $2.35,2.39$, and $2.40 \AA$ which give an average value of $2.37 \AA$. The remaining oxygens, $\mathrm{O}(7), \mathrm{O}(8), \mathrm{O}(9)$, and $\mathrm{O}(10)$, are only coordinated to one uranium atom. Here the $\mathrm{U}-\mathrm{O}$ distances vary between 2.37 and $2.53 \AA$. The $\mathrm{U}-\mathrm{Cl}$ distances are both $2.75 \AA$. The difference in size between chlorine and oxygen atoms, in addition to the stronger $\mathrm{U}-\mathrm{O}$ bonds within the uranyl groups, make the coordination polyhedra distorted. The two five-membered rings around the uranyl groups are coplanar. The least squares plane through all atoms in the dimer except the uranyl oxygens has the equation $-0.444 x-0.349 y-0.826 z+7.112=0$. None of the ten atoms deviates significantly from this plane (Fig. 4b). The uranyl groups are perpendicular to the plane; the calculated angles are $89.2^{\circ}$ and $89.9^{\circ}$.

The sevenfold coordination of uranium(VI) described here, with five atoms in the equatorial plane and the linear uranyl group perpendicular to the plane, has earlier been found in a great many compounds (Table 9). Comparison between the bond lengths given in Table 9 and those obtained in the present determination show good agreement with one exception. Such a high value as that of $2.53 \AA$ for the distance $\mathrm{U}(1)-\mathrm{O}(8)$ has only been found in a high-pressure form of $\mathrm{UO}_{3} \cdot{ }^{18}$

A review of the crystal structures, containing the uranyl group, known up to 1963 has been made by Evans. ${ }^{21} \mathrm{He}$ found that the uranyl group can coordinate four, five, or six oxygen atoms about its equator with a strong tendency to make these atoms coplanar. Fourfold and fivefold coordinations are geometrically stable. The oxygens can approach at an optimum distance in a plane without overcrowding. Stable planar sixfold coordination can occur when highly polarized, bidentate anionic groups donate the oxygen atoms which then can be drawn very close together. When oxygen atoms or hydroxide groups are brought into six-coordination around the uranyl group, they are forced out of the equatorial plane forming a puckered ring. Thus the stability of the structure tends to be reduced.

Crystal structure determinations published after 1963 show that some of the oxygens around the uranyl group can be replaced by other atoms, mainly chlorine atoms as in $\mathrm{Cs}_{x}\left(\mathrm{UO}_{2}\right) \mathrm{OCl}_{x}(x \approx 0.9),{ }^{16}$ the uranyl chlorides ${ }^{20}$ and the present compound, without changing the coordination polyhedron except for some distortion due to the difference in size between oxygen and chlorine atoms.

Table 10. Short $\mathrm{O}-\mathrm{O}(<3.0 \AA)$ and $\mathrm{O}-\mathrm{Cl}(<3.2 \AA)$ distances and corresponding angles

$\begin{array}{rcccc} & \begin{array}{c}\text { Distance } \\ (\AA)\end{array} & \begin{array}{c}\text { Letter in } \\ \text { Figs. } 1 \text { and } 2\end{array} & \begin{array}{c}\text { Angle } \\ \left({ }^{\circ}\right)\end{array} \\ \mathrm{O}(1)-\mathrm{O}(3) & 2.85(3) & \mathrm{C} & \mathrm{O}(3)-\mathrm{O}(1)-\mathrm{O}(7) & 111.8(10) \\ -\mathrm{O}(7) & 2.65(3) & \mathrm{E} & & \\ \mathrm{O}(2)-\mathrm{O}(6) & 2.93(3) & \mathrm{D} & \mathrm{O}(6)-\mathrm{O}(2)-\mathrm{O}(9) & 116.2(9) \\ -\mathrm{O}(9) & 2.58(3) & \mathrm{F} & & \\ \mathrm{O}(7)-\mathrm{Cl}(2) & 3.14(2) & \mathrm{A} & \mathrm{Cl}(2)-\mathrm{O}(7)-\mathrm{O}(1) & 108.7(8) \\ \mathrm{O}(9)-\mathrm{Cl}(1) & 3.15(2) & \mathrm{B} & \mathrm{Cl}(1)-\mathrm{O}(9)-\mathrm{O}(2) & 109.6(8) \\ & & & \text { Acta Chem. Scand. 23 (1969) No. 3 }\end{array}$


The dimers are arranged in sheets perpendicular to the $\vec{a}$ direction (Figs. 1 and 2). Since they are uncharged they must be held together by bonds of a different type than ionic, probably hydrogen bonds. In Table 10 short $\mathrm{O}-\mathrm{O}$ and $\mathrm{O}-\mathrm{Cl}$ distances are listed. These indicate the presence of hydrogen bonds. This assumption is supported by the size of the corresponding angles which are all rather close to the tetrahedron angle of $109^{\circ}$.

Four of the hydrogen bonds are extended mainly in the $\vec{c}$ direction, namely those joining an oxygen atom coordinated to only one uranium atom and a chlorine atom, $\mathrm{O}(7)-\mathrm{H} \cdots \mathrm{Cl}(2)$ (A in Figs. 1 and 2) and $\mathrm{O}(9)-\mathrm{H} \cdots \mathrm{Cl}(1)$ (B in Figs. 1 and 2), which are 3.14 and $3.16 \AA$, respectively, and those directed from a bridge oxygen atom toward a uranyl oxygen atom, $\mathrm{O}(1)-\mathrm{H} \cdots \mathrm{O}(3)$ $(\mathrm{C}$ in Fig. 1) and $\mathrm{O}(2)-\mathrm{H} \cdots \mathrm{O}(6)(\mathrm{D}$ in Fig. 1), whose lengths are 2.85 and 2.93 A, respectively.

The two remaining hydrogen bonds, which are the strongest ones, are

extended in the $\vec{b}$ direction joining a bridge oxygen atom and the same singly coordinated oxygen atom as above, $\mathrm{O}(7)-\mathrm{H} \cdots \mathrm{O}(1)(\mathrm{E}$ in Fig. 2) and $\mathrm{O}(9)-\mathrm{H} \cdots \mathrm{O}(2)(\mathrm{F}$ in Fig. 2). Their lengths are 2.65 and $2.58 \AA$, respectively.

In all known crystal structures of uranyl compounds it has been found that water molecules never form bridges between the uranyl groups. Accordingly, two hydroxide groups most probably constitute the bridge and the $O(7), O(8), O(9)$, and $O(10)$ atoms, which are bonded to only one uranium atom, belong to water molecules. The formula of the compound should then be written as $\left[\left(\mathrm{UO}_{2}\right)_{2}(\mathrm{OH})_{2} \mathrm{Cl}_{2}\left(\mathrm{H}_{2} \mathrm{O}\right)_{4}\right]$ to be in agreement with the structure.

All the hydrogen atoms of $\mathrm{O}(1), \mathrm{O}(2), \mathrm{O}(7)$, and $\mathrm{O}(9)$ have been indicated by the presence of hydrogen bonds. The remaining water oxygen atoms, $O(8)$ and $O(10)$, have in total four hydrogen atoms which are difficult to locate. No distances are short enough to give evidence for hydrogen bonds. Possibly the hydrogens are directed towards the chlorine atoms with the following distances
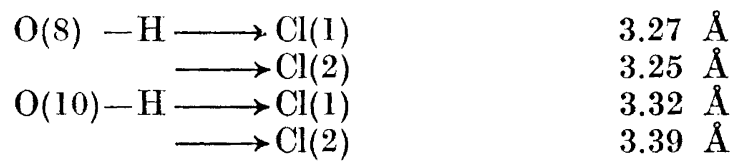

According to Pimentel and McClellan ${ }^{23}$ the upper limit of an $\mathrm{O}-\mathrm{H} \cdots \mathrm{Cl}$ bond is $3.25 \AA$. The angles $\mathrm{Cl}(1)-\mathrm{O}(8)-\mathrm{Cl}(2)$ and $\mathrm{Cl}(1)-\mathrm{O}(10)-\mathrm{Cl}(2)$ are $95^{\circ}$ and $76^{\circ}$, respectively, which is quite different from the tetrahedron angle.

The forces between the sheets of dimers must be rather weak as no short distances have been found indicating hydrogen bonds in the $\vec{a}$ direction. This is verified by the perfect cleavage of the crystals perpendicular to the $a$ axis.

In conclusion it can be said that the group $\left(\mathrm{UO}_{2}\right)_{2}(\mathrm{OH})_{2}$ which has been found in hydrolyzed solution seems to be retained on forming the crystalline state. Work is in progress on an investigation of hydrolyzed solutions of uranyl chloride using $\mathrm{X}$-ray diffraction in order to compare distances and coordination in the crystalline and liquid states.

Acta Chem. Scand. 23 (1969) No. 3 
Acknowledgements. I wish to thank Dr. Geory Johansson for his encouraging interest in this work and for valuable advice. Thanks are also due to Profs. Arne Magnéli and Lars Gunnar Sillén for criticism on the manuscript and to Civ.ing. Rune Söderquist for helpful discussions. The English text was corrected by Dr. George Baldwin.

\section{REFERENCES}

1. Martell, A. and Sillén, L. G. Tables of Stability Constants, Chem. Soc. Special Publication, No. 7, 2nd Ed., 1964.

2. Mylius, F. and Dietz, R. Ber. 34 (1901) 2774.

3. Peterson, S. J. Inorg. Nucl. Chem. 17 (1961) 135.

4. Hecht, F. and Donau, J. Anorganische Mikrogewichtsanalyse, Wien 1940, p. 205.

5. International Tables for X-ray Crystallography, Kynoch Press, Birmingham 1952, Vol I.

6. Wilson, A. J. C. Nature 150 (1942) 152.

7. Cromer, D. T. and Waber, J. T. Acta Cryst. 18 (1965) 104.

8. Berghuis, J., Haanappel, I. M., Potters, M., Loopstra, B. O., MacGillavry, C. H. and Veenendaal, A. L. Acta Cryst. 8 (1955) 478.

9. Cromer, D. T. Acta Cryst. 18 (1965) 17.

10. Hughes, E. W. J. Am. Chem. Soc. 63 (1941) 1737.

11. Lingafelter, E. C. and Donohue, J. Acta Cryst. 20 (1966) 321.

12. Zachariasen, W. H. Acta Cryst. 7 (1954) 783.

13. Appleman, D. E. Geol. Soc. Am. Bull. 68 (1956) 1696.

14. Smith, D. K., Jr., Gruner, J. W. and Lipscomb, W. N. Am. Mineralogist 42 (1957) 594.

15. Ross, M. and Evans, H. T., Jr. J. Inorg. Nucl. Chem. 15 (1960) 338.

16. Allpress, J. G. and Wadsley, A. D. Acta Cryst. 17 (1964) 41.

17. Appleman, D. E. and Evans, H. T., Jr. Am. Mineralogist 50 (1965) 825.

18. Siegel, S., Hoekstra, H. and Sherry, E. Acta Cryst. 20 (1966) 292.

19. Hall, D., Rae, A. D. and Waters, T. N. Acta Cryst. 22 (1967) 258.

20. Debets, P. C. Acta Cryst. B 24 (1968) 400.

21. Evans, H. T., Jr. Science 141 (1963) 154.

22. Pimentel, G. C. and McClellan, A. L. The Hydrogen Bond, San Francisco 1960.

Received July 19, 1968. 